\title{
Low star formation efficiency due to turbulent adiabatic compression in the Taffy bridge $e^{\star, \star \star, \star \star \star}$
}

\author{
B. Vollmer ${ }^{1}$, J. Braine ${ }^{2}$, B. Mazzilli-Ciraulo ${ }^{1,3}$, and B. Schneider ${ }^{1,4}$ \\ 1 Université de Strasbourg, CNRS, Observatoire astronomique de Strasbourg, UMR 7550, 67000 Strasbourg, France \\ e-mail: Bernd.Vollmer@astro.unistra.fr \\ ${ }^{2}$ Laboratoire d'astrophysique de Bordeaux, Univ. Bordeaux, CNRS, B18N, allée Geoffroy Saint-Hilaire, 33615 Pessac, France \\ 3 LERMA, Observatoire de Paris, PSL Research University, CNRS, Université de Sorbonne, UPMC, 75014 Paris, France \\ ${ }^{4}$ AIM, CEA, CNRS, Université Paris-Saclay, Université Paris Diderot, Sorbonne Paris Cité, 91191 Gif-sur-Yvette, France
}

Received 5 March 2020 / Accepted 15 January 2021

\begin{abstract}
The Taffy system (UGC 12914/15) consists of two massive spiral galaxies that had a head-on collision about 20 Myr ago. It represents an ideal laboratory for studying the reaction of the interstellar medium (ISM) to a high-speed $\left(\sim 1000 \mathrm{~km} \mathrm{~s}^{-1}\right)$ gas-gas collision. New sensitive, high-resolution $\left(2.7^{\prime \prime}\right.$ or $\left.\sim 800 \mathrm{pc}\right) \mathrm{CO}(1-0)$ observations of the Taffy system with the IRAM Plateau de Bure Interferometer $(\mathrm{PdBI})$ are presented. The total CO luminosity of the Taffy system detected with the PdBI is $L_{\mathrm{CO} \text {,tot }}=4.8 \times 10^{9} \mathrm{~K} \mathrm{~km} \mathrm{~s}^{-1} \mathrm{pc}^{2}, 60 \%$ of the CO luminosity found with the IRAM $30 \mathrm{~m}$ telescope. About $25 \%$ of the total interferometric CO luminosity stems from the bridge region. Assuming a Galactic $N\left(\mathrm{H}_{2}\right) / I_{\mathrm{CO}}$ conversion factor for the galactic disks and a third of this value for the bridge gas, about $10 \%$ of the molecular gas mass is located in the bridge region. The giant HII region close to UGC 12915 is located at the northern edge of the high-surface-brightness giant molecular cloud association (GMA), which has the highest velocity dispersion among the bridge GMAs. The bridge GMAs are clearly not virialized because of their high velocity dispersion. Three dynamical models are presented and while no single model reproduces all of the observed features, they are all present in at least one of the models. Most of the bridge gas detected in $\mathrm{CO}$ does not form stars. We suggest that turbulent adiabatic compression is responsible for the exceptionally high velocity dispersion of the molecular ISM and the suppression of star formation in the Taffy bridge. In this scenario the turbulent velocity dispersion of the largest eddies and turbulent substructures or clouds increase such that giant molecular clouds are no longer in global virial equilibrium. The increase in the virial parameter leads to a decrease in the star formation efficiency. The suppression of star formation caused by turbulent adiabatic compression was implemented in the dynamical simulations and decreased the star formation rate in the bridge region by $\sim 90 \%$. Most of the low-surface-density, CO-emitting gas will disperse without forming stars but some of the high-density gas will probably collapse and form dense star clusters, such as the luminous HII region close to UGC 12915. We suggest that globular clusters and super star clusters formed and still form through the gravitational collapse of gas previously compressed by turbulent adiabatic compression during galaxy interactions.
\end{abstract}

Key words. galaxies: interactions - galaxies: ISM

\section{Introduction}

Head-on collisions between spiral galaxies represent an ideal laboratory for studying the behavior of the interstellar medium (ISM) under extreme conditions. During the collision the interstellar media of both galactic disks collide, heat up, and exchange momentum. In merging galaxy pairs, an ISM-ISM collision occurs toward the end of the interaction process (see, e.g., Renaud et al. 2015 or Di Matteo et al. 2007). The Taffy system (UGC 12914/15; Fig. 1) is a special case because both spiral galaxies are particularly massive, were gas-rich before the collision, and collided at high speed $\left(\sim 1000 \mathrm{~km} \mathrm{~s}^{-1}\right.$; Condon et al. 1993, Vollmer et al. 2012a). We observe the galaxy pair about

\footnotetext{
* Movies associated to Figs. 8, B.3-B.5 are available at https://www . aanda.org

$\star \star$ The reduced datacube is only available at the CDS via anonymous ftp to cdsarc.u-strasbg.fr (130.79.128.5) or via http: //cdsarc.u-strasbg.fr/viz-bin/cat/J/A+A/647/A138

$\star \star \star$ Based on observations carried out with the IRAM Plateau de Bure Interferometer. IRAM is supported by INSU/CNRS (France), MPG (Germany) and IGN (Spain).
}

$20 \mathrm{Myr}$ after the impact that occurred in the plane of the sky. The transverse velocity difference at the present time is $650 \mathrm{~km} \mathrm{~s}^{-1}$.

The Taffy system attracted attention due to its strong radio synchrotron bridge (Condon et al. 1993), a very unusual feature. The bridge is HI-rich and was also subsequently found to be rich in molecular gas as well through $\mathrm{CO}$ observations (Gao et al. 2003; Braine et al. 2003). Dust appears to be underabundant with respect to gas in the bridge (Zink et al. 2000; Zhu et al. 2007), presumably due to grain ablation during the collision. The system contains about $1.5 \times 10^{10} M_{\odot}$ of $\mathrm{HI}$ and a similar quantity of molecular gas, depending on the $N\left(\mathrm{H}_{2}\right) / I_{\mathrm{CO}}$ conversion factor from $\mathrm{CO}$ emission to $\mathrm{H}_{2}$ column density. Some $10-20 \%$ of the gas is in the bridge, making it at least as rich in molecular gas as the entire Milky Way. The ionized gas is highly disturbed kinematically, with gas spread in two main filaments between the two galaxies. Hot, X-ray emitting gas that has presumably been shock-heated during the collision is also present in the bridge region (Appleton et al. 2015). This hot and tenuous gas is spatially more correlated with the low-density atomic gas and seems to avoid the high-density molecular gas.

The head-on collision of the Taffy system was simulated by Vollmer et al. (2012a) with a model that includes a collisionless 


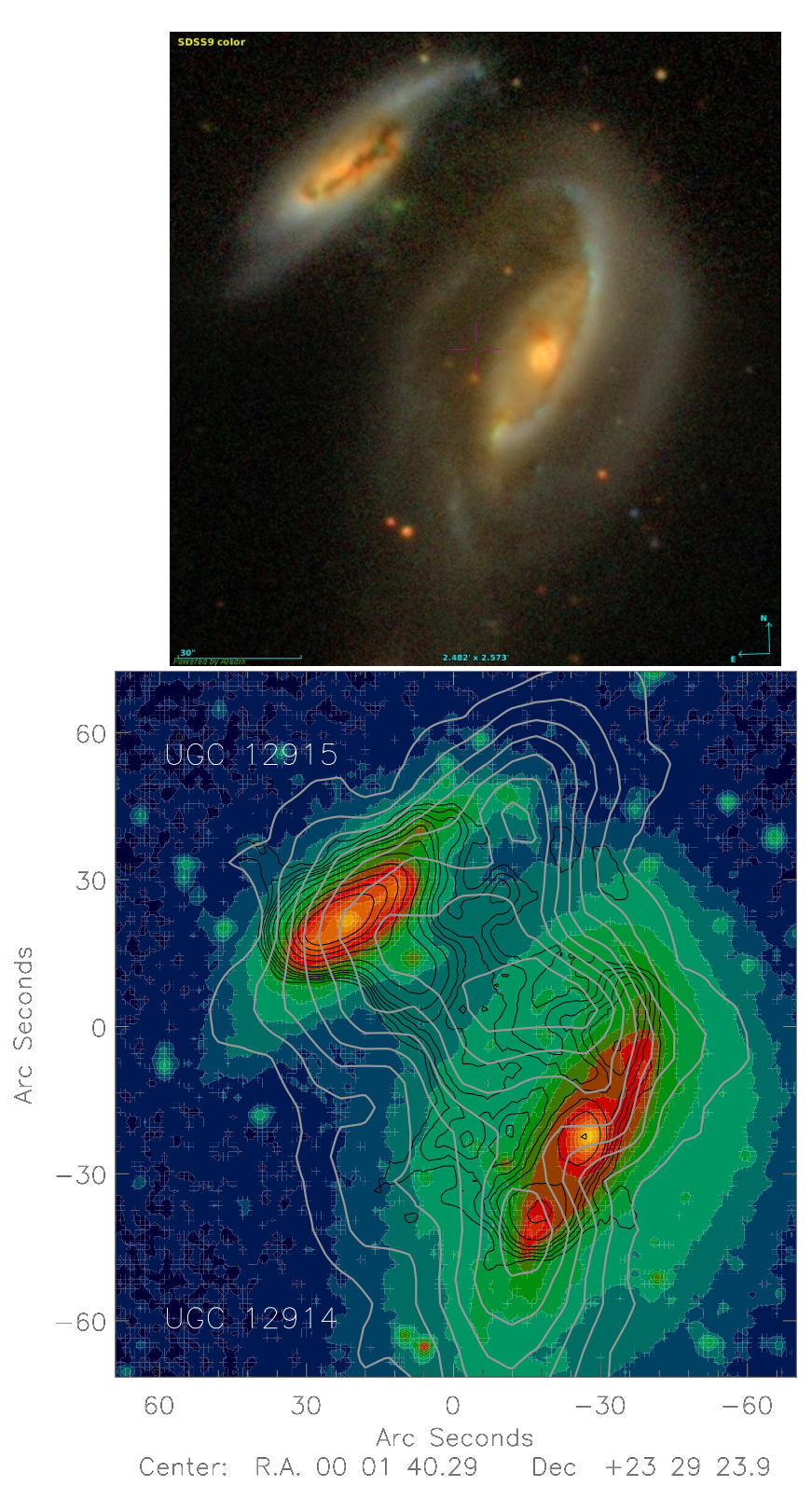

Fig. 1. Taffy system UGC 12914/15. Upper panel: SDSS color image. Lower panel: stellar content (Spitzer $3.6 \mu \mathrm{m}$ emission; denoted by the colors), Hi emission (light gray contours; Condon et al. 1993), and CO emission (black contours; Gao et al. 2003).

(halo and stellar particles) and a collisional (gas) component. A wealth of observational characteristics are available for the comparison with the simulations: a distorted stellar distribution, a prominent $\mathrm{HI}$ and $\mathrm{CO}$ gas bridge with large linewidths and $\mathrm{HI}$ double-line profiles, and a large-scale magnetic field with projected field vectors parallel to the bridge. Since these authors could not find a single simulation that reproduces all observed characteristics, they presented two "best-fit" simulations. The first simulation better reproduced the $\mathrm{HI}$ and $\mathrm{CO}$ line profiles of the bridge region (Braine et al. 2003), whereas the second simulation better reproduced the stellar distribution of UGC 12915 , the symmetric gas velocity fields of the galactic disks, the projected magnetic field vectors in the bridge region, and the distribution of the $6 \mathrm{~cm}$ polarized radio continuum emission (Condon et al. 1993). The stellar distribution of the model secondary galaxy is more distorted than that of UGC 12915. These models were successful in producing (1) the prominent HI and
$\mathrm{CO}$ gas bridge, (2) the offset of the $\mathrm{CO}$ emission to the south with respect to the HI emission in the bridge region, (3) the gas symmetric velocity fields in the galactic disks, (4) the isovelocity contours of the $\mathrm{CO}$ velocity field, which are parallel to the bridge, (5) the HI double-line profiles in the disk region, (6) the large gas linewidths $\left(100-200 \mathrm{~km} \mathrm{~s}^{-1}\right)$ in the bridge region, (7) the velocity separation between the double lines $\left(\sim 330 \mathrm{~km} \mathrm{~s}^{-1}\right)$, (8) the high field strength of the regular magnetic field in the bridge region, (9) the projected magnetic field vectors, which are parallel to the bridge, (10) the offset of the maximum of the $6 \mathrm{~cm}$ polarized radio continuum emission to the south of the bridge, and (11) and the strong total power emission from the disk. The structure of the model gas bridge was found to be bimodal: a dense $\left(\sim 0.01 M_{\odot} \mathrm{pc}^{-3}\right)$ component with a high velocity dispersion $>100 \mathrm{~km} \mathrm{~s}^{-1}$ and a less dense $\left(\sim 10^{-3} M_{\odot} \mathrm{pc}^{-3}\right)$ component with a smaller, but still high velocity dispersion of $\sim 50 \mathrm{~km} \mathrm{~s}^{-1}$. The synchrotron lifetime of relativistic electrons is only long enough to be consistent with the existence of the radio continuum bridge (Condon et al. 1993) for the less dense component. On the other hand, only the high-density gas undergoes a high enough mechanical energy input to produce the observed strong emission of warm $\mathrm{H}_{2}$ (Peterson et al. 2012).

The star formation efficiency of the molecular gas in the bridge region is at least two to three times smaller than that of the molecular gas located within the galactic disks (Vollmer et al. 2012a). There is one exception: A compact region of high star formation is located about $15^{\prime \prime}$, or $4.4 \mathrm{kpc}^{1}$, southwest of the center of UGC 12915. Despite low star formation rates (SFRs) in the bridge, the [C II] emission appears to be enhanced (Peterson et al. 2018), consistent with shock and turbulent gas heating (Joshi et al. 2019).

In this article, we present new high-resolution $\mathrm{CO}(1-0)$ observations of the Taffy system to better understand the distribution and kinematics of the dense molecular gas. In addition, we investigate why the star formation efficiency with respect to the molecular gas $\left(\mathrm{SFR} / M_{\mathrm{H}_{2}}\right)$ is so low in the gas bridge. To do so, the dynamical model of Vollmer et al. (2012a) was modified to include the effects of turbulent adiabatic compression and expansion. Both effects are able to temporarily suppress star formation in the dense gas.

\section{Observations}

Observations of the ${ }^{12} \mathrm{CO}(1-0)$ emission were carried out with the IRAM Plateau de Bure Interferometer (PdBI) in summer 2014 using all six antennas in C and D configuration. The system was covered by a mosaic of $11 \mathrm{PdBI}$ primary beams. Each position was observed during $55 \mathrm{~min}$. The bandpasses calibration was on 3C454.3 on May 30 and Nov. 21 and on 1749+096 on May 29. Phase and amplitude calibrations were performed on 2319+272 (every day), 0007+171 (21 Nov.), and 0006+243 (May 29 and 30). The absolute flux scale was checked on MWC 349 every day. A total bandwidth of $640 \mathrm{MHz}$ with a spectral resolution of $2.5 \mathrm{MHz}$ was used. We reach an rms of $\sim 5 \mathrm{mJy}$ in $6.5 \mathrm{~km} \mathrm{~s}^{-1}$ wide velocity channels. Applying robust weighting in the mapping process, a beam size of $2.7^{\prime \prime}(\sim 800 \mathrm{pc})$ was derived.

\section{Results}

The CPROPS (CloudPROPertieS) software (Rosolowsky \& Leroy 2006) was used to identify and measure the properties (size, flux, velocity dispersion) of molecular cloud associations

\footnotetext{
1 We use a distance of $60 \mathrm{Mpc}$ for the Taffy galaxy system.
} 
(GMAs) in the 2.7" datacube. The CPROPS program first assigns contiguous regions of the datacube to individual clouds and then computes the cloud properties (flux, radius, and velocity width) from the identified emission. The algorithm ignores clouds smaller than a resolution element and does not decompose clouds smaller than two resolution elements. We used the modified CLUMPFIND ${ }^{2}$ algorithm (ECLUMP) and required a peak of at least $1.5 \sigma$ in every distinct cloud and at least two channels. The CPROPS decomposition was used to produce the moment maps that are presented in Fig. 2. As a consistency check, we cleaned the datacube with a velocity channel width of $6.5 \mathrm{~km} \mathrm{~s}^{-1}$ by iteratively (i) boxcar averaging of each spectrum (width $=4$ channels), (ii) fitting Gaussians to the boxcar-averaged spectrum ( $v_{0}$ is the central velocity), (iii) all corresponding voxels in a 3D mask that are located between $v_{0}-F W H M$ and $v_{0}+F W H M$ are set to one, (iv) the Gaussian is subtracted from the boxcar-averaged spectrum, (v) the next Gaussian is fitted to the spectrum until its amplitude is smaller than $5 \sigma$ of the boxcar-averaged spectrum, (vi) the 3D mask is applied to the initial datacube. Moment maps were produced without clipping the datacube (Fig. A.1). The moment maps based on CPROPS and the "cleaned" moment maps are consistent, the former being deeper as expected from the lower CLUMPFIND limit of $1.5 \%$. The cloud or giant molecular cloud association (GMA) properties derived by CPROPS are shown in Table A.1.

\subsection{Moment maps}

The optical image of UGC 12915 (upper panel of Fig. 1) shows an asymmetric dust ridge or tilted ring visible in absorption and two symmetric stellar arms, the northern arm being brighter than the southern arm. The moment 0 map (Fig. 2) shows a bright, asymmetric, and twisted thin molecular disk rather than a tilted ring in UGC 12915 which corresponds to the asymmetric dust ridge. The surface brightness distribution along the major axis is asymmetric. The second brightest maximum in this disk corresponds to the galaxy center. The brightest maximum is located in the southeastern half of the disk. The northwestern half has a much lower surface brightness and is approximately twice as extended as the southeastern half of the disk. Moreover, the most northwestern part of UGC 12915's molecular disk is bent to the north, away from UGC 12914 and the bridge region.

The optical image of UGC 12914 (upper panel of Fig. 1) shows an inner lens structure with dust lanes and a much fainter outer double-ring structure. In addition, a stellar arm starts from the northern tip of the stellar lens structure and joins the eastern faint outer stellar ring. The $\mathrm{CO}$ emission distribution of UGC 12914 has three maxima along the major axis: the galaxy center (D1) and the two elongated structures at a distance of $\sim 20^{\prime \prime}$, or $5.8 \mathrm{kpc}$, from the center (D1 and D3). The latter structures correspond to the tips of the optical lens and are reminiscent of a limb-brightened molecular ring.

The northernmost part of UGC 12914's molecular disk is curved toward the bridge as is the stellar arm (upper panel of Fig. 1 and upper left panel of Fig. 7), suggesting this is a tidal effect. Whereas the western border of the CO distribution, which corresponds to the western dust lane within the optical lens structure of UGC 12914, is sharp, the eastern border is disrupted showing east-west filaments elongated into the bridge direction. These filaments are due to the ISM-ISM collision. The

\footnotetext{
2 For the CLUMPFIND algorithm, see Williams et al. (1994).
}

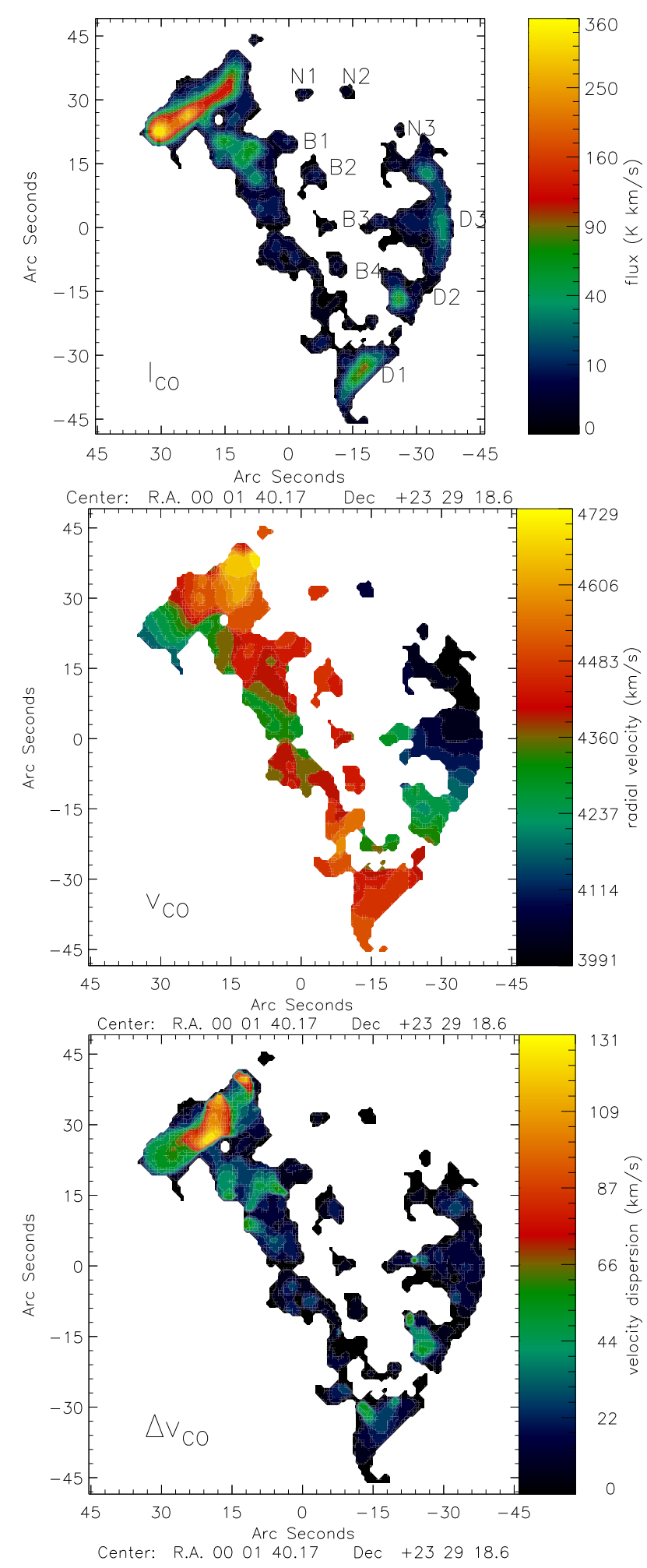

Fig. 2. $\mathrm{CO}(1-0)$ moment maps based on detections identified by CPROPS. Disk, bridge, and northern emission regions are labeled.

molecular gas bridge connecting the two galaxies has a width of $\sim 10^{\prime \prime}-30^{\prime \prime}$ or $\sim 3-9 \mathrm{kpc}$ and shows a maximum adjacent to the giant HII region close to UGC 12915 (upper panel of Fig. 3). It roughly connects the center of UGC 12915 and the southern CO maximum of UGC 12914. Four distinct CO clouds (B1-B4) are located parallel to the bridge to the west (upper panel of Fig. 2). Finally, three CO clouds (N1-N3) seem to connect the 


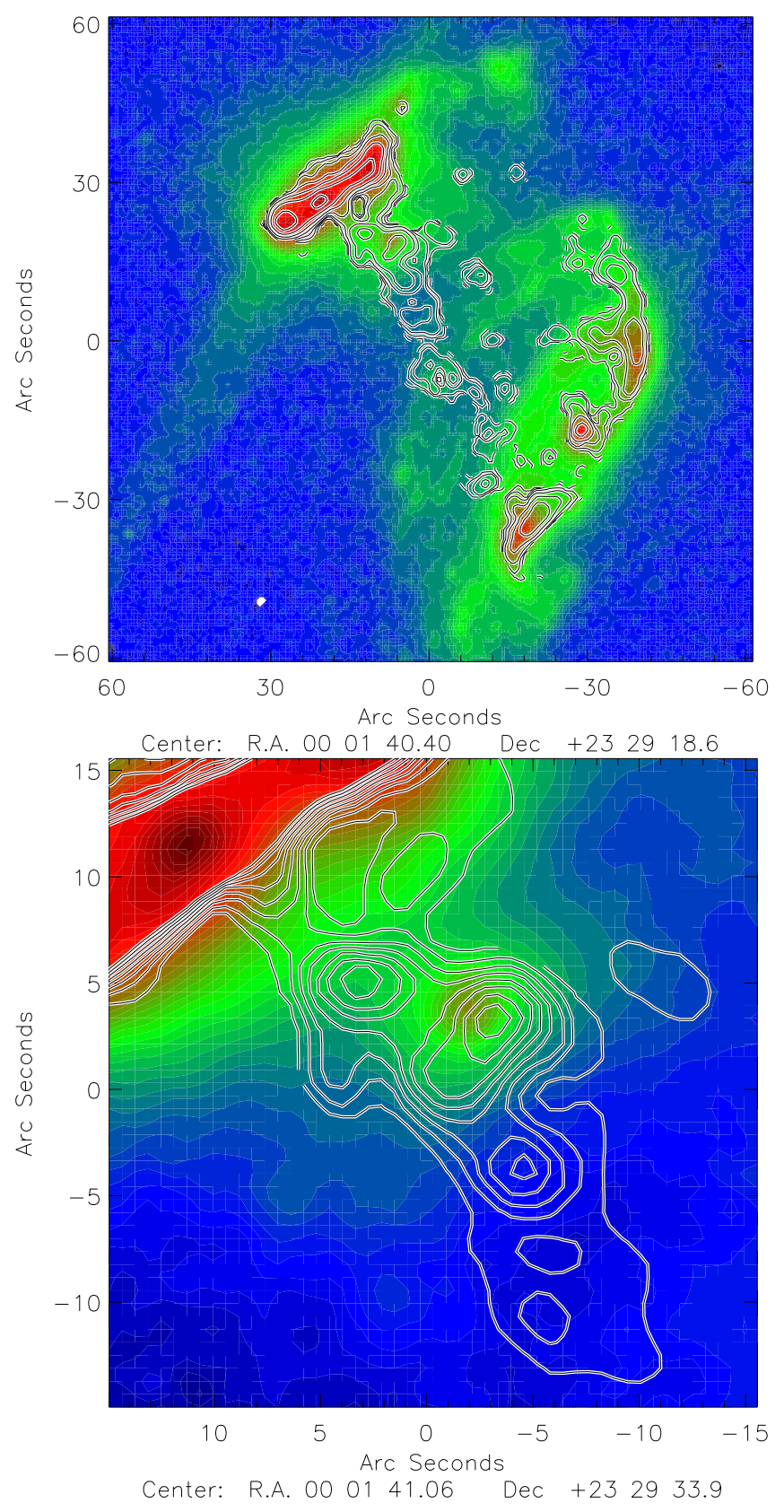

Fig. 3. $\mathrm{CO}(1-0)$ contours on the Spitzer $8 \mu \mathrm{m}$ PAH emission map. Upper panel: entire Taffy system. The stripe starting from the southern end of the disk of UGC 12915 is an image artifact. Contour levels are $(2,4,8,16,32,64,128,256) \mathrm{K} \mathrm{km} \mathrm{s}^{-1}$. Lower panel: zoom in on the compact extraplanar star-forming region south of UGC 12915. Contour levels are $(10,20,30,40,50,60,70,80) \mathrm{K} \mathrm{km} \mathrm{s}^{-1}$.

northern part of UGC 12914 and the northern part of the disk of UGC 12915.

The total CO luminosity of the Taffy system identified by CPROPS is $L_{\mathrm{CO} \text {,tot }}=4.8 \times 10^{9} \mathrm{~K} \mathrm{~km} \mathrm{~s}^{-1} \mathrm{pc}^{2}$. This represents $60 \%$ of the CO luminosity found by Braine et al. (2003) with the IRAM $30 \mathrm{~m}$ telescope. We divided the moment 0 into disk and bridge regions (Fig. A.2). The $\mathrm{CO}$ luminosity of the bridge is $L_{\mathrm{CO} \text {,bridge }}=1.2 \times 10^{9} \mathrm{~K} \mathrm{~km} \mathrm{~s}^{-1} \mathrm{pc}^{2}$. Thus, $25 \%$ of the total $\mathrm{CO}$ luminosity stems from the bridge region. Assuming a Galactic $N\left(\mathrm{H}_{2}\right) / I_{\mathrm{CO}}$ conversion factor for the galactic disks and a third of this value for the bridge gas, we obtain the following $\mathrm{H}_{2}$ masses: $M_{\mathrm{H}_{2} \text {,tot }}=1.7 \times 10^{10} M_{\odot}$ and $M_{\mathrm{H}_{2} \text {,bridge }}=1.7 \times 10^{9} M_{\odot}$. Thus,

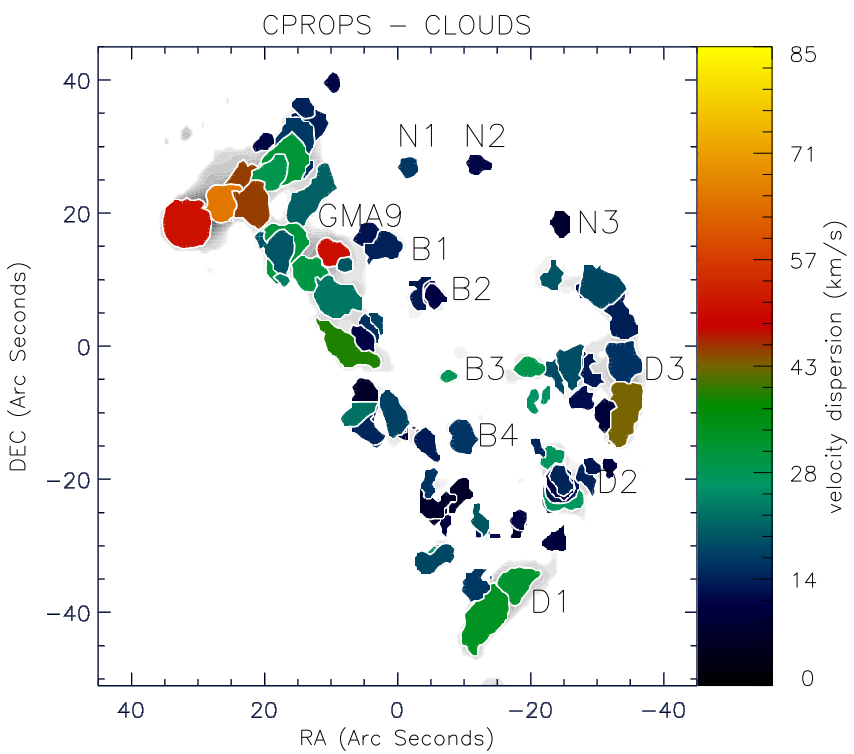

Fig. 4. Internal velocity dispersion of the CPROPS molecular clouds (color) on the moment 0 map (grayscale). Disk, bridge, and northern emission regions are labeled as in Fig. 2.

about $10 \%$ of the molecular gas mass is located in the bridge region.

An overlay with the Spitzer $8 \mu \mathrm{m}$ PAH emission map is shown in Fig. 3. Within the galactic disks, the $\mathrm{CO}(1-0)$ emission closely follows the high-surface-brightness $8 \mu \mathrm{m}$ emission. In the bridge region, there is dense gas traced by $\mathrm{CO}$ emission that is not forming stars, as shown by a lack of PAH emission, which is usually a tracer of star formation. This implies that the bulk of the bridge high-density gas does not form stars (see also Braine et al. 2003; Gao et al. 2003). The luminous compact extraplanar HII region south of UGC 12915 represents the exception to that rule. A close-up of the region (lower panel of Fig. 3) shows that the HII region does not coincide with, but is located at the northern edge of a high-surface-brightness GMA (GMA 9 in Table A.1). This GMA has the highest velocity dispersion of the bridge GMAs.

The velocity fields of UGC 12914 and UGC 12915 are dominated by rotation. The bridge shows a mixture of positive and negative radial velocities with respect to the systemic velocities of the galaxies $\left(4350 \mathrm{~km} \mathrm{~s}^{-1}\right)$. The region of high surface brightness close to UGC 12915 has an overall positive velocity with respect to the systemic velocity. The $\mathrm{CO}$ clouds aligned parallel to the bridge share this velocity range.

The internal velocity dispersions of the $\mathrm{CO}$ clouds derived by CPROPS are shown in Fig. 4. The velocity dispersion of the inner parts of the molecular disk in UGC 12914 is about $30 \mathrm{~km} \mathrm{~s}^{-1}$, roughly normal for an edge-on spiral galaxy at $800 \mathrm{pc}$ resolution. The highest velocity dispersions are found in the southeastern disk of UGC 12915 and its center. A cloud with a velocity dispersion of $\sim 50 \mathrm{~km} \mathrm{~s}^{-1}$ (GMA 9 in Table A.1 and Fig. 4) is found in the high-surface-brightness part of the bridge, close to the extraplanar HII region. Overall, the northern half of the gas bridge has significantly higher velocity dispersions than the southern half.

\subsection{Cloud properties}

We separated the CO clouds identified by CPROPS into disk and bridge clouds according to Fig. A.2. The resulting assignments 

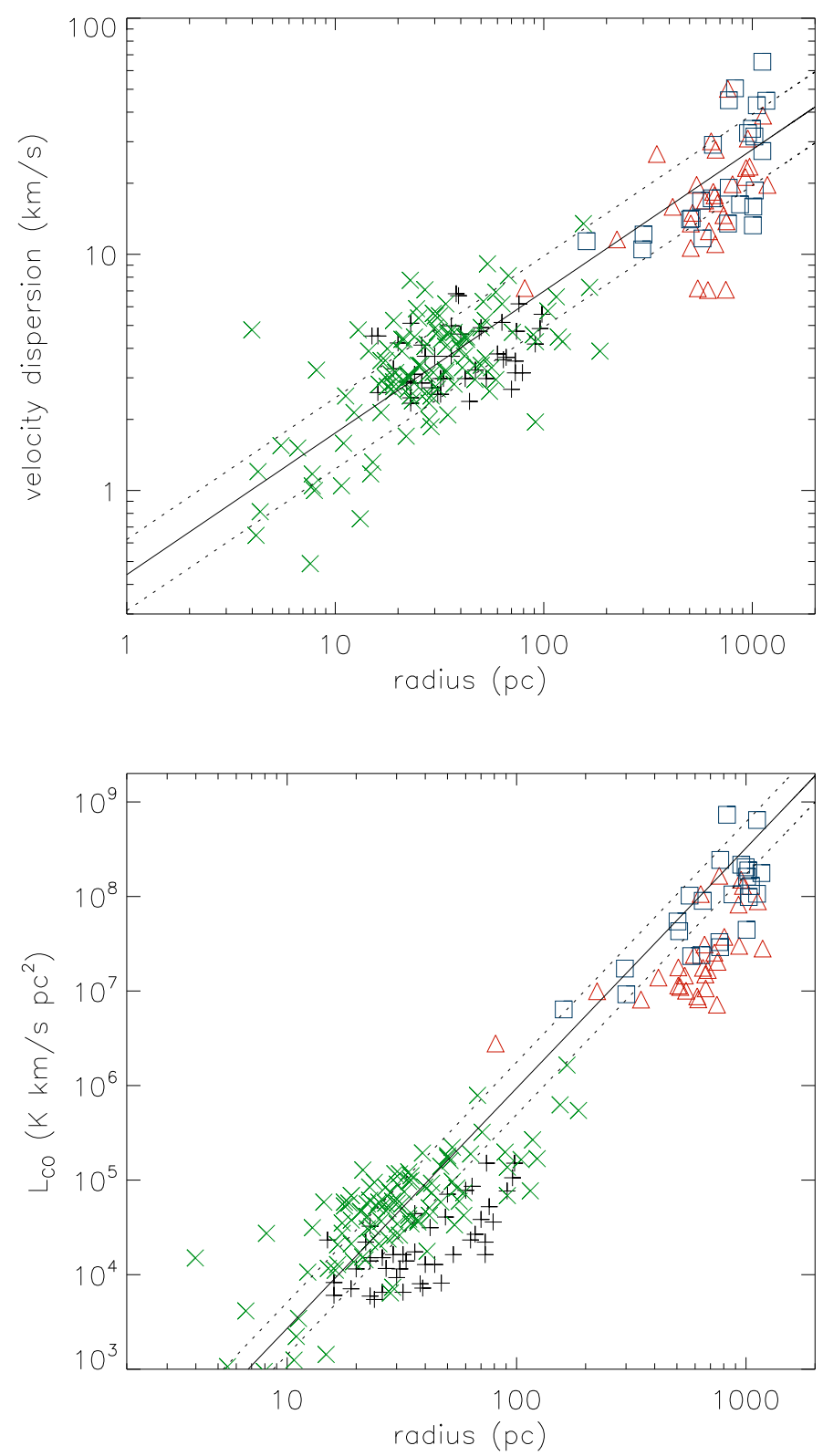

Fig. 5. Properties of CPROPS molecular entities (bridge: red triangles; galaxies: blue boxes) compared to data from Bolatto et al. (2008; green crosses) and Gratier et al. (2012; black pluses). Upper panel: velocity dispersion as a function radius. Lower panel: $\mathrm{CO}$ line flux as a function of radius. The lines correspond to the relations determined by Bolatto et al. (2008).

are given in Table A.1. The cloud properties are compared to those of extragalactic GMAs from Bolatto et al. (2008) and those of M 33 derived by Gratier et al. (2012) in Fig. 5. With a resolution of $2.7^{\prime \prime}$ or $800 \mathrm{pc}$ we can only detect GMAs. It is remarkable that the GMAs in the disk and bridge regions follow, as the molecular clouds in M 33, the size-linewidth relation established by Bolatto et al. (2008) that is valid for extragalactic and Galactic molecular clouds. The scatter around the relation is also comparable to that of Bolatto et al. (2008) and Gratier et al. (2012). It is especially surprising that the disk GMAs follow the relation because a significant fraction of their linewidth is expected to be caused by large-scale motions, namely rotation and noncircular motions. The offset between the velocity dispersion determined by CPROPS and that predicted by the size-linewidth relation is presented in Fig. 6. For clarity we only colored GMAs whose

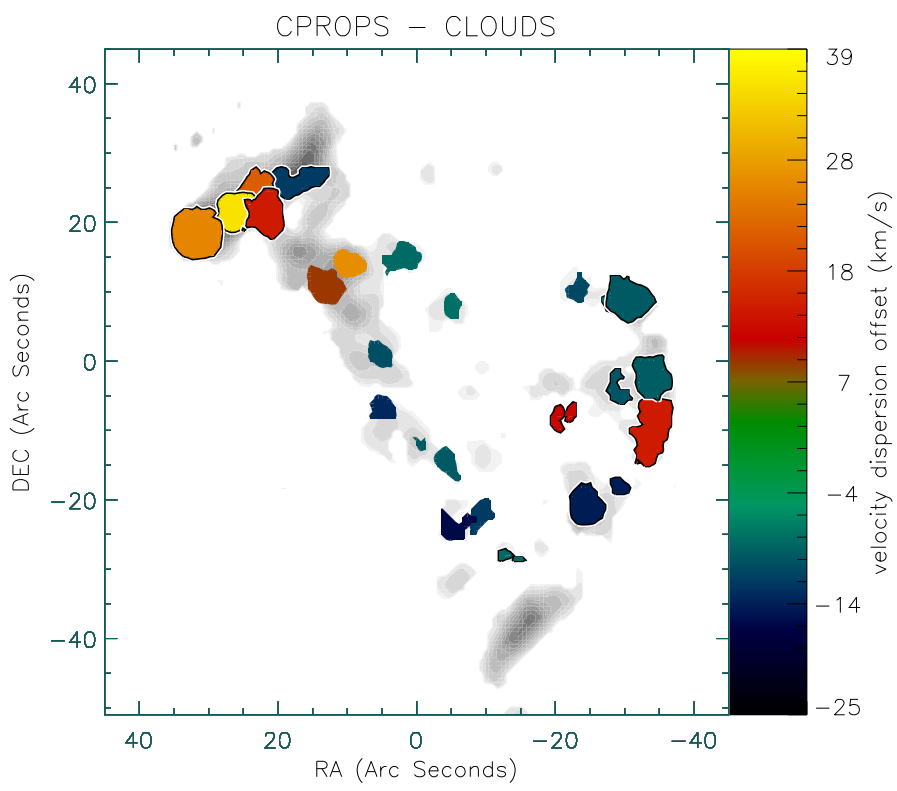

Fig. 6. Velocity dispersion offset of CPROPS molecular clouds with respect to the relation for extragalactic GMCs found by Bolatto et al. (2008). Only GMAs with linewidth outside $1 \sigma$ of the size-linewidth relation shown in Fig. 5 are colored. The disk GMAs are marked with a black contour.

linewidths are outside $1 \sigma$ of the size-linewidth relation. Two regions with exceptionally high linewidths stand out from this figure: the southeastern half of the UGC12915 disk and the region around the extraplanar HII region close to UGC12915.

The size-luminosity relation of the GMAs in the bridge and disk regions is different. Whereas the clouds in the galactic disks follow the relation established by Bolatto et al. (2008), the majority of the bridge clouds show about three times lower CO luminosity than expected from the relation. The molecular clouds in M 33 are also CO-underluminous by about a factor of two. Gratier et al. (2012) argued that this is due to a two times higher $N\left(\mathrm{H}_{2}\right) / I_{\mathrm{CO}}$ conversion factor. For the Taffy bridge region Braine et al. (2003) excluded a higher $N\left(\mathrm{H}_{2}\right) / I_{\mathrm{CO}}$ conversion factor based on their ${ }^{13} \mathrm{CO}$ measurements. On the contrary, Braine et al. (2003) and $\mathrm{Zhu}$ et al. (2007) argued that the $N\left(\mathrm{H}_{2}\right) / I_{\mathrm{CO}}$ conversion factor is several times lower in the bridge than in the galactic disk.

\section{Comparison to dynamical models}

Vollmer et al. (2012a) calculated 17 models of head-on collisions of two gas-rich spiral galaxies. To the two "best-fit" models presented by these authors we added a third model with a higher velocity between the two galaxies. The maximum impact velocity is $1200 \mathrm{~km} \mathrm{~s}^{-1}$ and the transverse velocity difference at the present time is $\sim 900 \mathrm{~km} \mathrm{~s}^{-1}$ versus $\sim 700 \mathrm{~km} \mathrm{~s}^{-1}$ for the previous simulations. We call this new simulation "sim19fast". Because of the high time resolution of our simulations the cloud collisions are well resolved even for this enormous impact velocity. The system is observed at the same lapse of time (20 Myr) after impact as the two simulations in Vollmer et al. (2012a).

\subsection{Moment maps}

The comparison between the observed surface brightness distribution and the model moment 0 maps is shown in Fig. 7. 

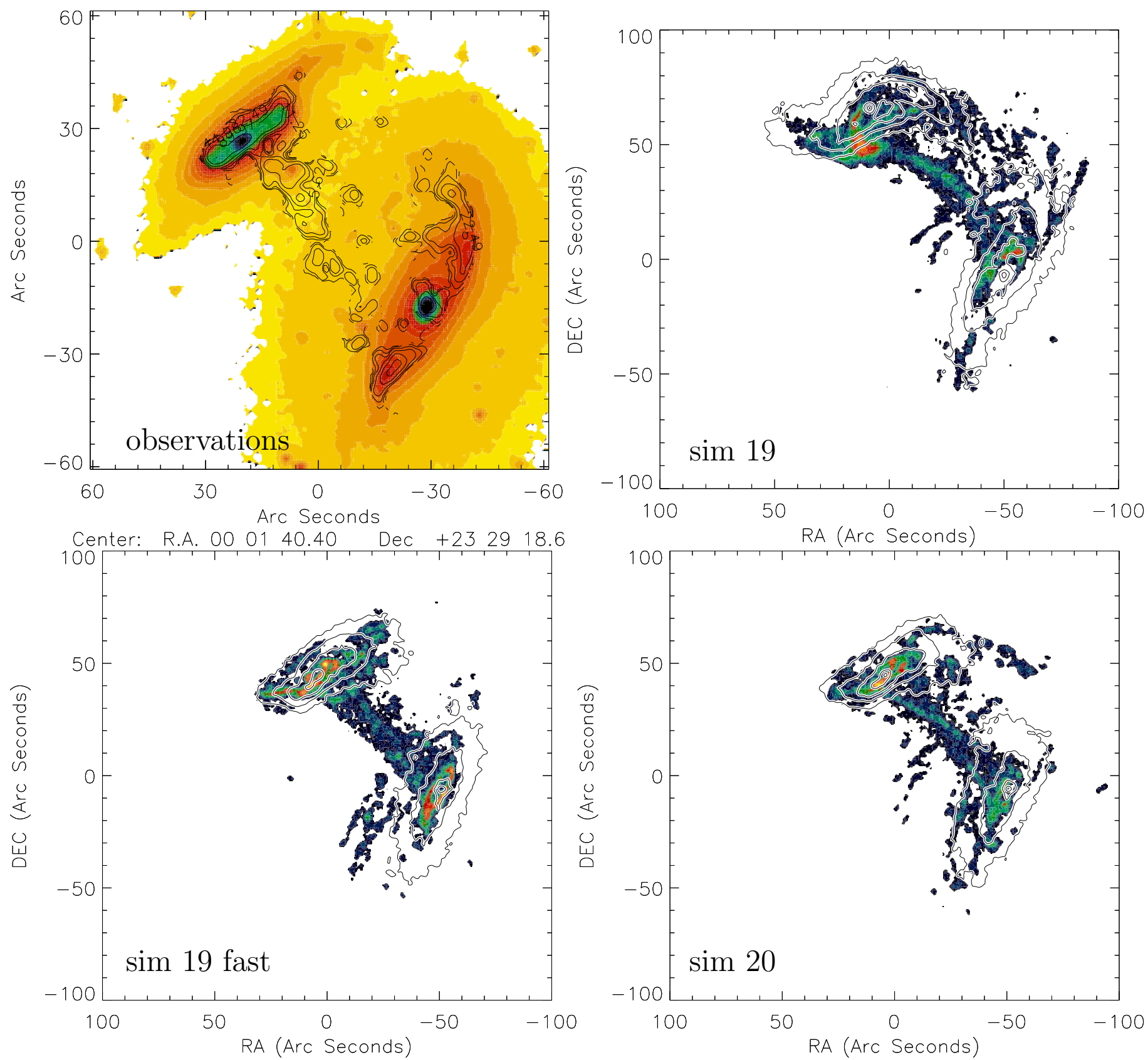

Fig. 7. $\mathrm{CO}(1-0)$ moment 0 maps together with the model $\mathrm{H}_{2}$ moment 0 maps. Upper left panel: PdBI observations (contour) together with the Spitzer $3.6 \mu \mathrm{m}$ map. The contour levels are $(0.35,0.7,1.4,2.8,5.6,11.2,22.5,45.0,90.0,180.0,360.0) \mathrm{K} \mathrm{km} \mathrm{s}^{-1}$. In the other panels, the contours show stellar distribution and the colors indicate molecular gas distribution. The color stretch is the same as the contours of the upper left panel.

All three simulations develop a gas-rich bridge and show the observed sharp western border of gas distribution of UGC 12914 which is mainly a tidal feature. As already stated in Vollmer et al. (2012a), none of the models reproduce the detailed morphology of the system. Whereas the model bridge starts close to the center of the northern galaxy, as is observed, it joins the southern galaxy also close to its center. In the observations the bridge joins the disk of UGC 12914 further to the south. The edge-on projection of UGC 12915 is better reproduced by sim20. On the other hand, the east-west asymmetry of its surface brightness is better reproduced by $\operatorname{sim} 19$ and sim 19 fast. Contrary to observations, all models show a second bridge filament to the west of the main bridge. This filament is brightest in sim19fast. The northern part of the disk of UGC 12914 with its filaments pointing toward UGC 12915 is reproduced by sim19fast and to a much lesser degree by $\operatorname{sim} 19$. It is not reproduced by $\operatorname{sim} 20$, because the northern galaxy passed through the southern galaxy at this location, removing all gas there. Only in model sim19fast, the gas near the northern galaxy is much denser than that close to the southern galaxy, as is observed.

The comparison between the observed velocity field and the model moment 1 maps is shown in Fig. B.1. The velocity field of UGC 12914 is reasonably reproduced by sim19fast and to a lesser degree by sim19, whereas that of UGC 12915 is best reproduced by $\operatorname{sim} 20$. The velocity field of the bridge with its positive and negative velocities with respect to the systemic velocity is best reproduced by $\operatorname{sim} 20$ and to a much lesser degree by $\operatorname{sim} 19$ fast. The model secondary bridge filaments to the north 
B. Vollmer et al.: Low star formation efficiency due to turbulent adiabatic compression in the Taffy bridge

Table 1. Comparison between our $\mathrm{CO}(1-0)$ and GALEX FUV observations and the models.

\begin{tabular}{lccc}
\hline \hline Feature & sim19 & sim19fast & sim20 \\
\hline Gas morphology of UGC 12915 & - & - & + \\
Gas morphology of UGC 12914 & + & + & $\sim$ \\
Morphology of the gas bridge & $\sim$ & $\sim$ & $\sim$ \\
Velocity field of UGC 12915 & $\sim$ & $\sim$ & + \\
Velocity field of UGC 12914 & + & + & + \\
Velocity field of the gas bridge & $\sim$ & $\sim$ & + \\
Velocity dispersion of the gas bridge & - & + & - \\
Global FUV morphology & $\sim$ & + & $\sim$ \\
Large-scale magnetic field $^{(a)}$ & + & & + \\
\hline
\end{tabular}

Notes. ${ }^{(a)}$ Based on the results of Vollmer et al. (2012a).

with their high velocities with respect to the systemic velocity are not observed. We conclude that a single model among our limited set of simulations (see Vollmer et al. 2012a) is not able to reproduce the observed characteristics of the Taffy system. However, almost all characteristics can be found in one of the three models. In many ways, this is to be expected as the initial gas distribution is not known. The advantages and disadvantages of the models are summarized in Table 1.

The comparison between the observed velocity dispersion and the model moment 2 maps is shown in Fig. B.2. None of the models reproduce the extremely high velocity dispersion in the disk of UGC 12915. In sim19 and sim 20 the regions of highest velocity dispersion are located close to the southern galaxy. In $\operatorname{sim} 20$ another region of high velocity dispersion is located in the middle of the bridge where the two bridge filaments cross. Only sim19fast shows a velocity dispersion in the bridge region close to the northern galaxy that is comparable to the observed velocity dispersion. We conclude that $\operatorname{sim} 19$ fast is in rough agreement with the observed distribution of the velocity dispersion in the bridge.

\section{2. $3 D$ visualization of the datacubes}

To appreciate the full wealth of information provided by the datacubes, we decided to compare the observed and the model datacubes by means of a 3D visualization. Here, we provide four different views of the datacubes rendered at the same given intensity (Figs. 8 and B.3-B.5).

The total linewidth of UGC 12914 is significantly smaller than that of the southern model galaxy. This can be due to an overestimated model inclination angle or an overestimated rotation velocity of the southern model galaxy. The bridge region of high surface brightness and intensity near UGC 12915 has an inverted V-shape in the projection of Fig. 8. Moreover, it is confined to a relatively narrow velocity range around the systemic radial velocity. A filament of low surface brightness and intensity emanating from this region smoothly joins the high radial velocity part of the disk of UGC 12914 . The only model that reproduces these features is $\operatorname{sim} 20$. However, the region of high intensities is further away from the northern galaxy than is observed for UGC 12915. All models show emission emanating from the sides of highest and lowest radial velocities in the northern galaxy. These features are not observed in UGC 12915. The velocity structure of the southern galaxy is rather well reproduced. As already mentioned, only the northern part of the gas disk of the southern galaxy is missing in the model $\operatorname{sim} 20$, because the impact entirely removed the gas there. Inspection of Figs. B.3-B.5 corroborates these conclusions.

\section{Star formation suppression caused by turbulent adiabatic compression}

What do the Circumnuclear Disk in the Galactic Center, a thick obscuring AGN torus, the ram-pressure stripped and tidally distorted Virgo spiral galaxy NGC 4438, Stephan's Quintet, and the Taffy galaxies all have in common? At the first glance, all these systems are very different. First of all, the spatial scales and timescales differ enormously. The CND and AGN tori have spatial extents of about $10 \mathrm{pc}$ and rotation timescales of $10^{4} \mathrm{yr}$, whereas the relevant scales and timescales in NGC 4438, Stephan's Quintet, and the Taffy galaxies are on the order of tens of kiloparsecs and $100 \mathrm{Myr}$. The common property of all these systems is that they are undergoing gas-gas collisions with high energy injection rates. In these collisions, one gaseous body is the turbulent clumpy multiphase ISM, while the other can be of different mean density and temperature (for example ISM, intragroup or intracluster gas): NGC 4438 is affected by ongoing ram pressure caused by its rapid motion through the Virgo intracluster medium (Vollmer et al. 2005, 2009), and the intragroup gas of the Stephan's Quintet is compressed by a highvelocity intruder galaxy (Appleton et al. 2017). We suggest that the common theme of all these gas-gas interactions is adiabatic large-scale compression of the ISM leading to an increase in the turbulent velocity dispersion of the gas (Robertson \& Goldreich 2012; Mandal et al. 2020).

It is generally assumed that within the disks of isolated galaxies turbulence is driven by energy injection through stellar feedback (SN explosions). In an equilibrium state a balance between turbulent pressure and gravity is reached leading to a global virial equilibrium state of the GMCs (Heyer et al. 2009). If the energy injection through large-scale gas compression exceeds that of stellar feedback deduced via the SFR, the velocity dispersion of the largest eddies is expected to increase. In this case, we presume that the velocity dispersion of the turbulent substructures or clouds also increases (Fig. 2 of Mandal et al. 2020). Such clouds were observed in the Galactic Center region by Oka et al. (1998, 2001). As a result, these GMCs will no longer be in global virial equilibrium. Oka et al. (2001) argued that the high virial parameters $\left(\alpha_{\mathrm{vir}}=5 \sigma_{\mathrm{cl}}^{2} R_{\mathrm{cl}} /\left(G M_{\mathrm{cl}}\right)\right.$, where $\sigma_{\mathrm{cl}}, R_{\mathrm{cl}}$, and $M_{\mathrm{cl}}$ are the cloud $1 \mathrm{D}$ velocity dispersion, radius, and mass) of the Galactic Center GMCs may explain the paucity of star formation activity in this region. Indeed, analytical and numerical models of turbulent star-forming gas clouds predict a decreasing star formation efficiency per free fall timescale with the virial parameter of a GMC (Federrath \& Klessen 2012; Padoan et al. 2012, 2017).

Following Robertson \& Goldreich (2012) and Mandal et al. (2020), we expect turbulent adiabatic heating, for example, an increase in the turbulent velocity dispersion due to the $\mathrm{pdV}$ work, to occur if the timescale of large-scale gas compression

$t_{\text {comp }}=\rho /(\mathrm{d} \rho / \mathrm{d} t)$

is smaller than the dissipation timescale of turbulence $t_{\text {diss }}$. From the dynamical simulations of Vollmer et al. (2012a) we derived a compression timescale within the bridge of $t_{\text {comp }} \lesssim$ $10 \mathrm{Myr}$ (Fig. C.4). The driving length in the bridge is somewhere between the average cloud size $\left(l_{\mathrm{cl}} \sim 1 \mathrm{kpc}\right.$ from Table A.1) and the filament width $(\sim 3 \mathrm{kpc})$, considerably longer than for GMCs 


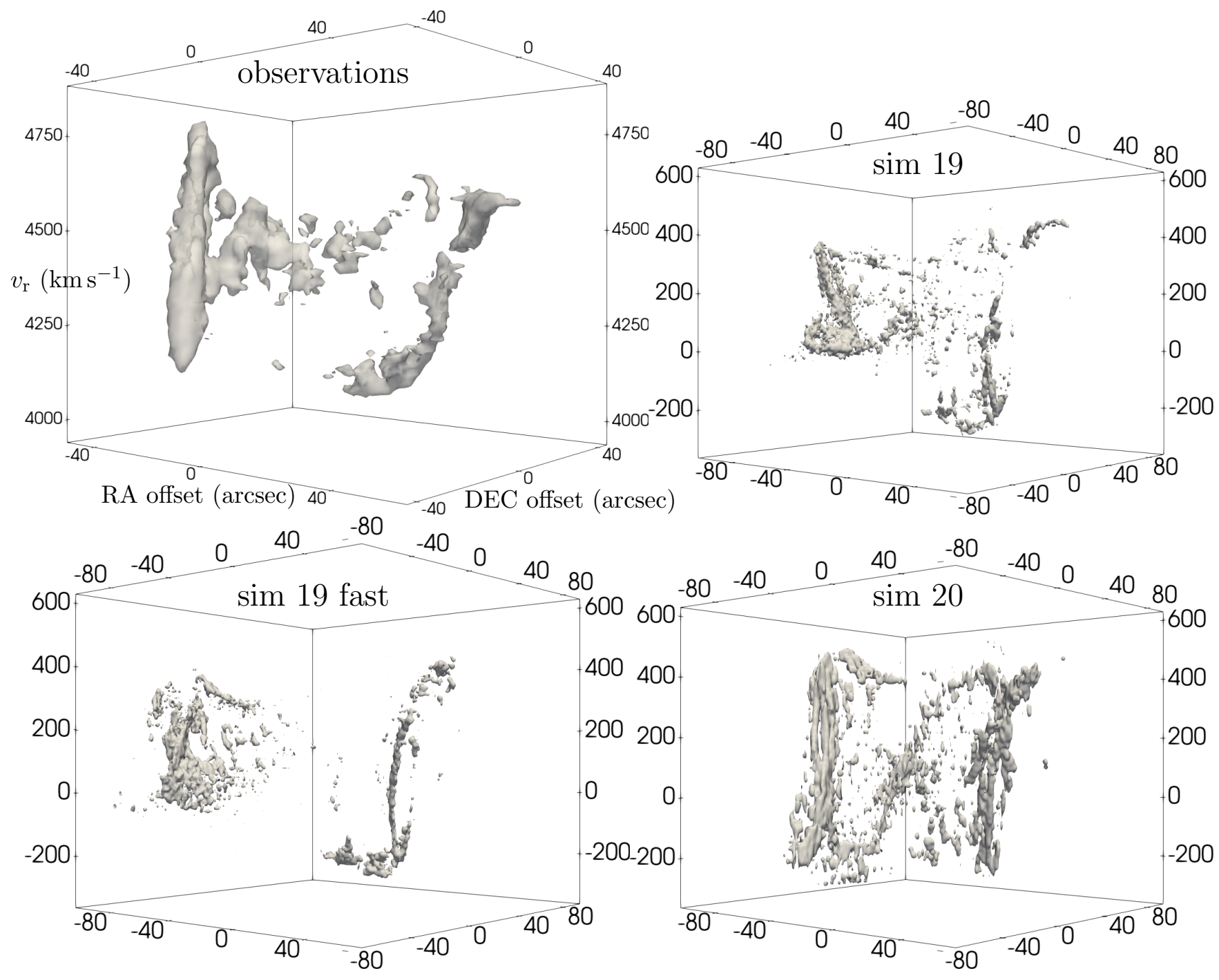

Fig. 8. First $3 \mathrm{D}$ view of the observed $\mathrm{CO}(1-0)$ datacube and the model $\mathrm{H}_{2}$ datacubes. These views correspond to a position-velocity diagram. The axis labels are only shown for the observations. For a better understanding of these views, three 3D animations of the rotating datacube are available online (taffy_cube3D_z.gif, taffy_cube3D_z1.gif, and taffy_cube3D_x.gif).

in an unperturbed disk. The crossing time is then approximately $t_{\text {cross }} \sim 2 \mathrm{kpc} / 50 \mathrm{~km} \mathrm{~s}^{-1} \sim 40 \mathrm{Myr}$ and this can be taken as $t_{\mathrm{diss}}$. A detailed comparison between the compression and dissipation timescales of the model is given in Appendix C. The $t_{\text {comp }}$ is significantly smaller than $t_{\text {diss }}$ in the bridge but not in the galaxies. Thus, we expect high virial parameters and weak star formation in the bridge gas. Adiabatic compression and its effect on star formation are included in the dynamical model and the results are compared to observations in Appendix C.

\section{Discussion}

The shorter the timescale, the more important the process is. In this work, we compare the dissipation timescale, a few megayears as given in Eq. (C.8) which assumes energy injection via star formation, to the compression timescale (Eq. refeq:tcomp1). In a disk environment, there is little compression, that is $\mathrm{d} \rho / \mathrm{d} t$ is small, and hence the compression time is long, such that dissipation is the dominant process (Fig. C.3). During the Taffy collision, and afterward in the bridge region, extremely strong shocks are present (as witnessed by the $\mathrm{H}_{2}$ emission observed by Peterson et al. 2018) and $\mathrm{d} \rho / \mathrm{d} t$ becomes enormous, and thus $t_{\text {comp }}$ short (Fig. C.4). Furthermore, the dissipation timescale $\left(l_{\text {driv }} / v_{\text {turb }}\right)$ in the bridge is higher due to the much longer driving scale (Appendix C.4). These two factors result in $t_{\text {comp }}<t_{\text {diss. }}$. The injected energy cannot be evacuated and this largely suppresses the star formation in the bridge.

A single model among our limited set of simulations cannot reproduce all observed characteristics of the Taffy system. However, all characteristics are present in one of the models (Table 1). The models sim 19 and sim19fast fail to reproduce the gas morphology of UGC 12915, because the model inclination is significantly lower than the observed edge-on projection. Since the parameter space for the head-on collision of both galaxies is vast, we did not try to search for better initial conditions than those found in Vollmer et al. (2012a) and thus a better reproduction of the Taffy system. We could show that the observed detailed velocity structure of the gas bridge can be well reproduced by one of our models (sim20; Fig. B.1). The observed north-south surface brightness gradient of the gas bridge and the increased velocity dispersion of its high-surface-brightness part can be reproduced by model sim19fast (Fig. 7). We are thus confident that such a model is in principle possible to account for all observed characteristics (Table 1). 
Based on our models, we could show that a high-velocity head-on encounter can lead to a significant fraction of the bridge gas undergoing turbulent adiabatic compression $20 \mathrm{Myr}$ after impact. We claim that the absence of star formation in bridge regions is due to turbulent adiabatic compression where the turbulent velocity dispersion of the largest eddies increases. It is expected that the velocity dispersions of the turbulent substructures or clouds increase such that GMCs are no longer in global virial equilibrium. The increase in the virial parameter leads to a decrease in the star formation efficiency per free fall timescale in the turbulent ISM (Federrath \& Klessen 2012; Padoan et al. 2012,2017 ) and thus to the suppression of star formation.

Relating the Virial mass of a gas cloud to its CO-derived mass yields

$\sigma=\sqrt{\pi / 5 G R \sigma}$

where $R$ and $\Sigma$ are the radius and surface density of the cloud. For the disk clouds we applied the Galactic $N\left(\mathrm{H}_{2}\right) / I_{\mathrm{CO}}$ conversion factor, for the bridge clouds a three times lower $N\left(\mathrm{H}_{2}\right) / I_{\mathrm{CO}}$ conversion factor. The resulting relation is shown in Fig. 9.

Whereas the Virial mass of the molecular clouds from Bolatto et al. (2008) and Gratier et al. (2012) are higher than the gas masses derived from the $\mathrm{CO}$ luminosities, the Virial masses of the Taffy disk GMAs are consistent with the gas masses derived from the $\mathrm{CO}$ luminosities. Again, this is surprising because a significant fraction of their linewidth is expected to be caused by large-scale motions, namely rotation and noncircular motions. Therefore, one should not expect a correlation and the one we found is most probably coincidental.

Based on the comparison between simulations and observations, Vollmer et al. (2012a) concluded that the bridge extent along the line-of-sight is small compared to its extent in the plane of the sky and the dominant component of the gas velocities follows the bridge geometry with small line-of-sight gradients. Applying a Virial analysis and assuming a $N\left(\mathrm{H}_{2}\right) / I_{\mathrm{CO}}$ conversion factor of a third of the Galactic Value, these GMAs have masses well below the Virial mass. They are thus far from being selfgravitating. The same behavior is observed in the $\Sigma R-\sigma$ relation (Eq. (2)) and is expected in a scenario where the turbulent ISM is compressed adiabatically.

The gas in the bridge region has different phases: The molecular gas is mainly arranged in a filament with a width of $\sim 3 \mathrm{kpc}$, the maximum of the neutral hydrogen emission distribution is shifted to the northwest of the CO filament (Condon et al. 1993), there are two distinct filaments of ionized gas (Fig. 6 of Joshi et al. 2019) and the X-ray emission (Appleton et al. 2015) both of which are also shifted to the northwest of the CO filament. Thus, the dense molecular gas avoids the other gas phases, especially the diffuse warm and hot phases. Could it be that the secondary gas tail that is present in all simulations (Fig. 7) is not molecular, but atomic and/or ionized? Based on the FUV image (Fig. C.10), we argue that the HI maximum stems from gas that belongs to UGC 12914 than to the bridge (see sim19 in Fig. 7). The ionized gas is prominent at negative velocities with respect to the systemic velocity. It thus belongs kinematically more to UGC 12914. The morphology of the hot X-ray emitting gas is reminiscent of the gas distribution of the northern bridge filament in sim 19 and sim19fast. In our simulations this gas has mostly positive velocities with respect to the systemic velocity. It is thus unlikely that the observed ionized gas coincides with the northern bridge filament. The observed HI in the bridge region (Condon et al. 1993) has a double line structure, as the ionized gas. At low velocities $\left(4060-4320 \mathrm{~km} \mathrm{~s}^{-2}\right.$ )
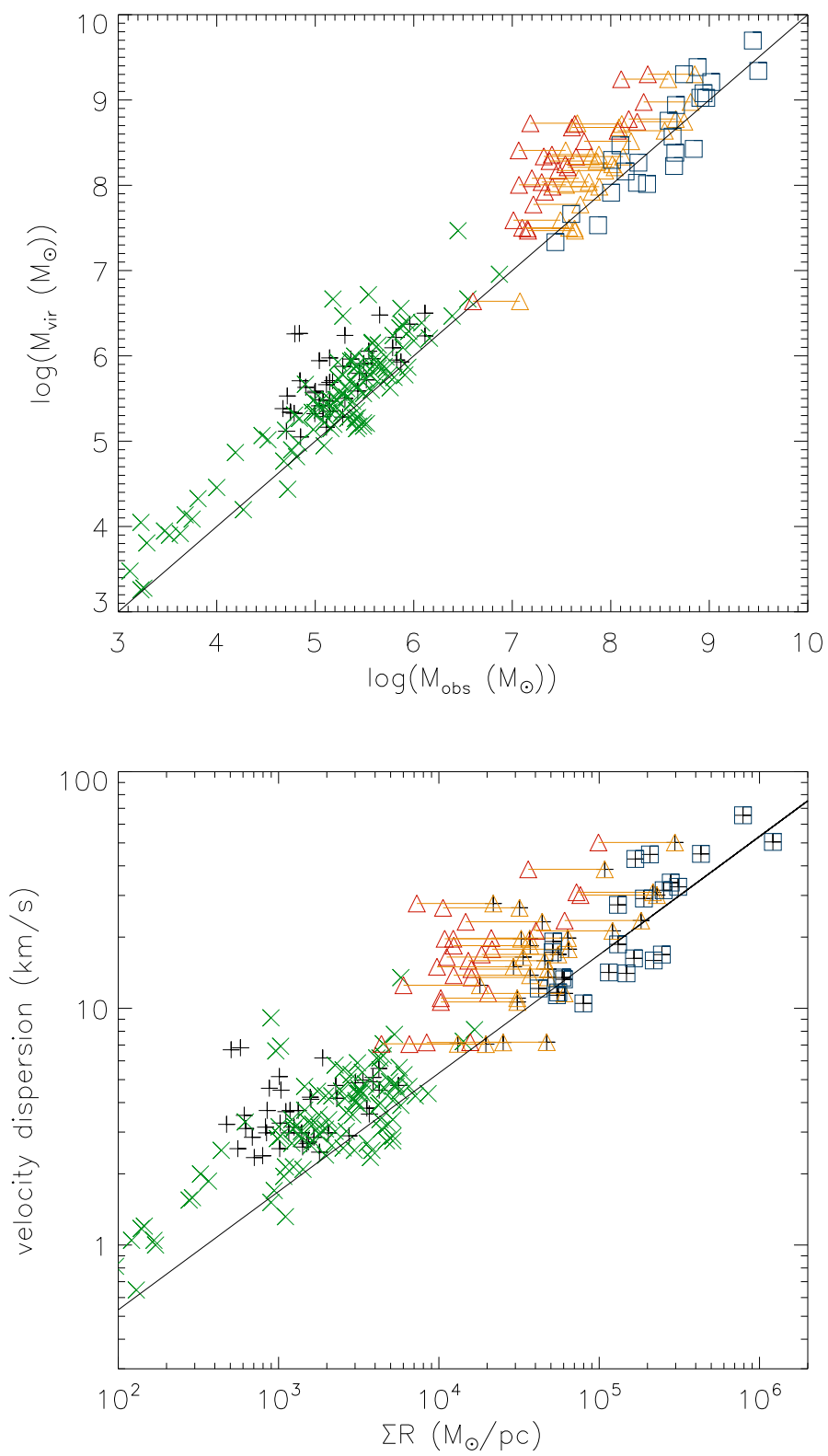

Fig. 9. CO cloud properties. Upper panel: cloud virial mass as a function of the gas mass derived from the CO luminosity. We applied a $N\left(\mathrm{H}_{2}\right) / I_{\mathrm{CO}}$ conversion factor that is one third of the Galactic value to the bridge clouds (red triangles). The orange triangles correspond to a Galactic $N\left(\mathrm{H}_{2}\right) / I_{\mathrm{CO}}$ conversion factor. Lower panel: cloud velocity dispersion as a function of the product of size and mass surface density. CPROPS clouds (bridge: red triangles; galaxies: blue boxes) are compared to data from Bolatto et al. (2008; green crosses) and Gratier et al. (2012; black pluses). The orange triangles correspond to a Galactic $N\left(\mathrm{H}_{2}\right) / I_{\mathrm{CO}}$ conversion factor. The solid line corresponds to Eq. (2).

the HI channel maps show a northwest-southeast velocity gradient. At high velocities $\left(4440-4570 \mathrm{~km} \mathrm{~s}^{-1}\right)$ there seems to be a southwest-northeast gradient present. The low-velocity part of the HI emission belongs to UGC 12914, whereas the highvelocity part belongs to UGC 12915.

What is the fate of the bridge gas? Will the high-surfacedensity bridge region close to UGC 12915 collapse and form stars or will it expand and disperse? We think that most of the low-surface-density, CO-emitting gas will disperse without forming stars. On the other hand, the high-density gas will probably have a different fate. It is remarkable that the luminous 
extraplanar HII region close to UGC 12915 does not coincide with a bridge GMA (but there is a GMA close to it; lower panel of Fig. 3). This implies that the gas cloud(s) from which the HII region has formed has already been disrupted by stellar feedback (stellar wind and supernova explosions). For this process we offer the following explanation: The compression timescale is proportional to the gas density (Eq. (C.11)), where the dissipation timescale is proportional to the square root of the density (Eq. (C.8)). At the beginning of the phase of adiabatic compression the gas density is not too high permitting $t_{\text {comp }}<t_{\text {diss. }}$. During the phase of adiabatic compression the gas density increases until $t_{\text {comp }}>t_{\text {diss }}$ and the region collapses and forms stars. On

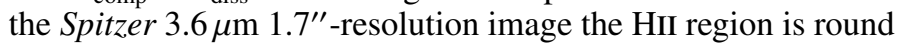
and has FWHM of $5^{\prime \prime}$ or $\sim 1.5 \mathrm{kpc}$. This is about the same size as GMA 9 (Table A.1). Compared to the extreme molecular cloud in the Antennae system (Johnson et al. 2015), GMA 9 has a mass that is about ten times higher mass $\left(\sim 2 \times 10^{8} M_{\odot}\right.$ assuming a $N\left(\mathrm{H}_{2}\right) / I_{\mathrm{CO}}$ conversion factor which is one third of the Galactic value), but a comparable velocity dispersion. The size of the Antennae cloud is only $24 \mathrm{pc}$. This implies that GMA 9 will certainly be resolved into several distinct clouds. We can only speculate that single massive high-velocity dispersion molecular clouds collapsed due to their high density and formed the HII region composed of several dense star clusters. High-resolution ALMA CO observations (Appleton et al., in prep.) will give further insight into the formation scenario of this atypical HII region.

We suggest that star clusters with extreme stellar densities $\left(\gtrsim 10^{4}\right.$ stars $\left.\mathrm{pc}^{-3}\right)$, such as globular clusters and super star clusters (O'Connell et al. 1994), formed and still form through the gravitational collapse of gas previously compressed by turbulent adiabatic compression during galaxy interactions. During the compression phase the cloud accumulates mass and increases its velocity dispersion. The high velocity dispersion prevents collapse but once the critical density reached the turbulent energy is dissipated rapidly and the cloud collapses and forms an extremely dense and massive star cluster.

This scenario probably applies to the extragalactic HII region close to UGC 12915 (lower panel of Fig 3): The Pa- $\alpha$ emission of the HII region detected in the HST NICMOS ${ }^{3}$ F190N filter (upper panel of Fig. 10) has a complex structure within a circular region of $\sim 600 \mathrm{pc}$ diameter: a central prominent compact source with a FWHM of $0.4^{\prime \prime}=120 \mathrm{pc}$ with a northern extension and three fainter compact sources of about the same size. In the F187N off-band filter (lower panel of Fig. 10) only the prominent compact source and a second compact source in the northern ionized extension are visible. The size of the compact source is in excess but comparable to the size of the largest super star cluster in the Antennae galaxies (SSC_B: FWHM of $1^{\prime \prime}=95 \mathrm{pc}$ and mass of $5 \times 10^{6} M_{\odot}$; Gilbert \& Graham 2007). The F187N emission is either dominated by massive $\mathrm{O}$ stars if the super star clusters are younger than $\sim 8 \mathrm{Myr}$ or by red supergiants if they are older. The maximum age of the clusters is given by the time since the interaction $20 \mathrm{Myr}$. Based on these findings we suggest that super star clusters were and maybe still are formed within the bridge HII region close to UGC 12915.

\section{Conclusions}

The Taffy system is composed of two massive spiral galaxies that had a head-on collision about $20 \mathrm{Myr}$ ago. We present new high-resolution $\left(\sim 2.7^{\prime \prime}\right) \mathrm{CO}(1-0)$ observations with the

\footnotetext{
Retrieved from the MAST HLA database.
}

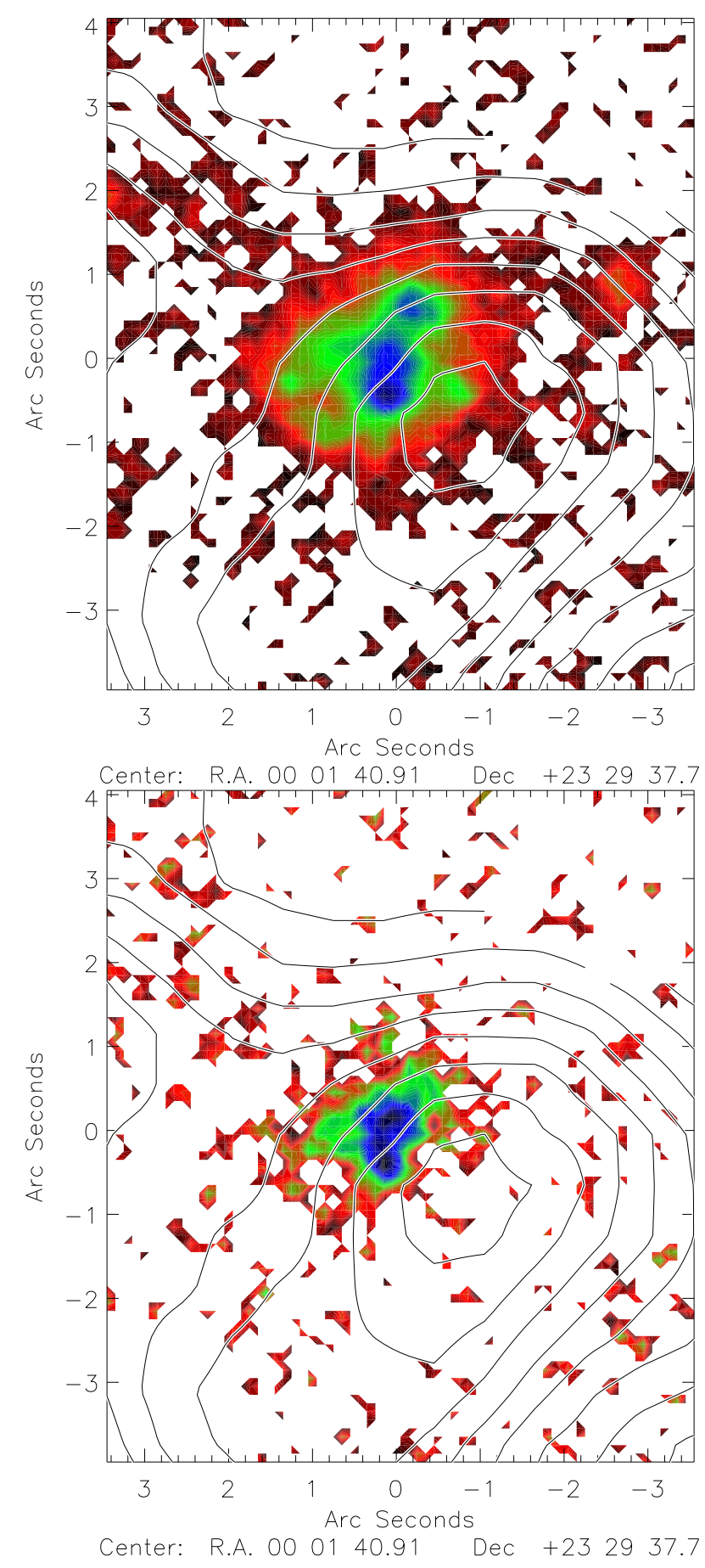

Fig. 10. Close-up of the giant bridge HII region, $\mathrm{CO}(1-0)$ contours on the HST NICMOS F190N (Pa- $\alpha$; upper panel) and F187N (off-band; lower panel) image (PropID 11080, PI: D. Calzetti). The HST astrometry was approximately aligned with the Spitzer astrometry. Contour levels are $(10,20,30,40,50,60,70,80) \mathrm{K} \mathrm{km} \mathrm{s}^{-1}$.

PdBI. An rms of $\sim 5 \mathrm{mJy}$ in a $6.5 \mathrm{~km} \mathrm{~s}^{-1}$ channel was reached by our observations. The CPROPS software (Rosolowsky \& Leroy 2006) was used to identify and measure the properties of GMAs. The detected CO luminosity of the Taffy system is $L_{\mathrm{CO} \text {,tot }}=4.8 \times 10^{9} \mathrm{~K} \mathrm{~km} \mathrm{~s}^{-1} \mathrm{pc}^{2}$. We divided the CO intensity map into disk and bridge regions (Fig. A.2). The CO luminosity of the bridge is $L_{\mathrm{CO} \text {,bridge }}=1.2 \times 10^{9} \mathrm{~K} \mathrm{~km} \mathrm{~s}^{-1} \mathrm{pc}^{2}, 25 \%$ of the total $\mathrm{CO}$ luminosity. Assuming a Galactic $N\left(\mathrm{H}_{2}\right) / I_{\mathrm{CO}}$ conversion factor for the galactic disks and a third of this value for 
the bridge gas, we obtain $\mathrm{H}_{2}$ masses of $M_{\mathrm{H}_{2} \text {,tot }}=1.7 \times 10^{10} M_{\odot}$ and $M_{\mathrm{H}_{2} \text {,tot }}=1.7 \times 10^{9} M_{\odot}$. Thus, about $10 \%$ of the molecular gas mass is located in the bridge region.

The bulk of the bridge high-density gas does not form stars (Braine et al. 2003; Gao et al. 2003). The luminous extraplanar HII region south of UGC 12915 represents the exception to that rule. A close-up of the region (lower panel of Fig. 3) shows that the HII region does not coincide with, but is located at the northern edge of a high-surface-brightness GMA (GMA 9 in Table A.1) with a flux of $1.7 \times 10^{8} \mathrm{~K} \mathrm{~km} \mathrm{~s}^{-1} \mathrm{pc}^{2}$ and a velocity dispersion of $50 \mathrm{~km} \mathrm{~s}^{-1}$ ). This GMA has the highest velocity dispersion of the bridge GMAs.

We separated the CO clouds identified by CPROPS into disk and bridge clouds. It is remarkable that the GMAs in the disk and bridge regions approximately follow the size-linewidth relation established by Bolatto et al. (2008) for extragalactic and Galactic molecular clouds. The scatter around the relation is also comparable to that of Bolatto et al. (2008) and Gratier et al. (2012). On the other hand, the size-luminosity relations of the GMAs in the bridge and disk regions are different: The bridge GMAs have lower luminosities for their sizes than the disk GMAs, and the bridge GMAs are clearly not virialized.

The $\mathrm{CO}(1-0)$ observations were compared to the dynamical models of Vollmer et al. (2012a) together with a new simulation. None of the simulations reproduce all observed features of the Taffy system. However, all characteristics can be found in one of the three models. Table 1 lists the features reproduced (or not) by each of the models.

Rapid turbulent adiabatic compression induced by the $\sim 1000 \mathrm{~km} \mathrm{~s}^{-1}$ collision could explain the high velocity dispersions and the subsequent suppression of star formation (Federrath \& Klessen 2012; Padoan et al. 2012, 2017) in the Taffy bridge. In this scenario the turbulent velocity dispersions of the largest eddies and their substructures or clouds increase such that GMCs are no longer in global virial equilibrium.

The suppression of star formation caused by turbulent adiabatic compression was implemented in the dynamical simulations: Once the gas compression timescale is shorter than the turbulent dissipation timescale, star formation is suppressed. This mechanism decreased the model star formation in the bridge region by a factor of about three to five, consistent with observations.

The bulk of the bridge molecular gas is not gravitationally bound and will disperse. The densest regions will probably become self-gravitating and form stars as in the giant bridge HII region. Because of their enhanced velocity dispersion these regions are much denser and more massive than common galactic GMCs. This mechanism could explain the extreme stellar densities in globular clusters and super star clusters (O'Connell et al. 1994), as observed in the Antennae.

Acknowledgements. We would like to thank the IRAM staff for the help observing the Taffy system with the PdBI. Based on observations made with the
NASA/ESA Hubble Space Telescope, and obtained from the Hubble Legacy Archive, which is a collaboration between the Space Telescope Science Institute (STScI/NASA), the Space Telescope European Coordinating Facility (STECF/ESA) and the Canadian Astronomy Data Centre (CADC/NRC/CSA). We thank Dominique Aubert for the creation of the $3 \mathrm{D}$ views.

\section{References}

Appleton, P. N., Lanz, L., Bitsakis, T., et al. 2015, ApJ, 812, 118 Appleton, P. N., Guillard, P., Togi, A., et al. 2017, ApJ, 836, 76 Bolatto, A. D., Leroy, A. K., Rosolowsky, E., et al. 2008, ApJ, 686, 948 Braine, J., Davoust, E., Zhu, M., et al. 2003, A\&A, 408, L13 Combes, F., \& Gerin, M. 1985, A\&A, 150, 327

Condon, J. J., Helou, G., Sanders, D. B., \& Soifer, B. T. 1993, AJ, 105, 1730

Di Matteo, P., Combes, F., Melchior, A.-L., et al. 2007, A\&A, 468, 61

Elmegreen, B. G., Elmegreen, D. M., \& Leitner, S. N. 2003, ApJ, 590, 271

Faucher-Giguère, C.-A., Quataert, E., \& Hopkins, P. F. 2013, MNRAS, 433, 1970

Federrath, C., \& Klessen, R. S. 2012, ApJ, 761, 156

Gao, Y., Zhu, M., \& Seaquist, E. R. 2003, AJ, 126, 2171

Gratier, P., Braine, J., Rodriguez-Fernandez, N. J, et al. 2008, A\&A, 542, A108

Gilbert, A. M., \& Graham, J. R. 2007, ApJ, 668, 168

Harwit, M., Houck, J. R., Soifer, B. T., et al. 1987, ApJ, 315, 28

Heyer, M., Krawczyk, C., Duval, J., et al. 2009, ApJ, 699, 1092

Johnson, K. E., Leroy, A. K., Indebetouw, R., et al. 2015, ApJ, 806, 35

Joshi, B. A., Appleton, P. N., Blanc, G. A., et al. 2019, ApJ, 878, 161

Krumholz, M. R., \& McKee, C. F. 2005, ApJ, 630, 250

Krumholz, M. R., Dekel, A., \& McKee, C. F. 2012, ApJ, 745, 69

Krumholz, M. R., Burkhart, B., Forbes, J. C., et al. 2018, MNRAS, 477, 2716

Leitherer, C., Schaerer, D., Goldader, J. D., et al. 1999, ApJS, 123, 3

Leroy, A. K., Walter, F., Brinks, E., et al. 2008, AJ, 136, 2782

Leroy, A. K., Schinnerer, E., Hughes, A., et al. 2017, ApJ, 846, 71

Mandal, A., Federrath, C., \& Körtgen, B. 2020, MNRAS, 493, 3098

Oka, T., Hasegawa, T., Hayashi, M., et al. 1998, ApJ, 493, 730

Oka, T., Hasegawa, T., Sato, F., et al. 2001, ApJ, 562, 348

O'Connell, R. W., Gallagher, J. S., \& Hunter, D. A. 1994, ApJ, 433, 65

Ostriker, E. C., \& Shetty, R. 2011, ApJ, 731, 41

Padoan, P., Haugbølle, T., \& Nordlund, Å. 2012, ApJ, 759, L27

Padoan, P., Haugbølle, T., Nordlund, A., et al. 2017, ApJ, 840, 48

Peterson, B. W., Appleton, P. N., Helou, G., et al. 2012, ApJ, 751, 11

Peterson, B. W., Appleton, P. N., Bitsakis, T., et al. 2018, ApJ, 855, 141

Renaud, F., Bournaud, F., \& Duc, P.-A. 2015, MNRAS, 446, 2038

Robertson, B., \& Goldreich, P. 2012, ApJ, 750, L31

Rosolowsky, E., \& Leroy, A. 2006, PASP, 118, 590

Springel, V., Yoshida, N., \& White, S. D. M. 2001, New Astron., 6, 79

Vollmer, B., \& Beckert, T. 2003, A\&A, 404, 21

Vollmer, B., \& Leroy, A. K. 2011, AJ, 141, 24

Vollmer, B., Braine, J., Combes, F., \& Sofue, Y. 2005, A\&A, 441, 473

Vollmer, B., Soida, M., Chung, A., et al. 2009, A\&A, 496, 669

Vollmer, B., Braine, J., \& Soida, M. 2012a, A\&A, 547, A39

Vollmer, B., Soida, M., Braine, J., et al. 2012b, A\&A, 537, A143

Vollmer, B., Gratier, P., Braine, J., et al. 2017, A\&A, 602, A51

Wiegel, W. 1994, Diploma Thesis, University of Heidelberg

Williams, J. P., de Geus, E. J., \& Blitz, L. 1994, ApJ, 428, 693

Wolfire, M. G., McKee, C. F., Hollenbach, D., et al. 2003, ApJ, 587, 278

Yeager, T. R., \& Struck, C. 2019, MNRAS, 486, 2660

Yeager, T. R., \& Struck, C. 2020, MNRAS, 492, 4892

Zamora-Avilés, M., \& Vázquez-Semadeni, E. 2014, ApJ, 793, 84

Zhu, M., Gao, Y., Seaquist, E. R., \& Dunne, L. 2007, AJ, 134, 118

Zink, E. C., Lester, D. F., Doppmann, G., \& Harvey, P. M. 2000, ApJS, 131, 413 
Appendix A: Moment maps, CPROPS results, and bridge separation
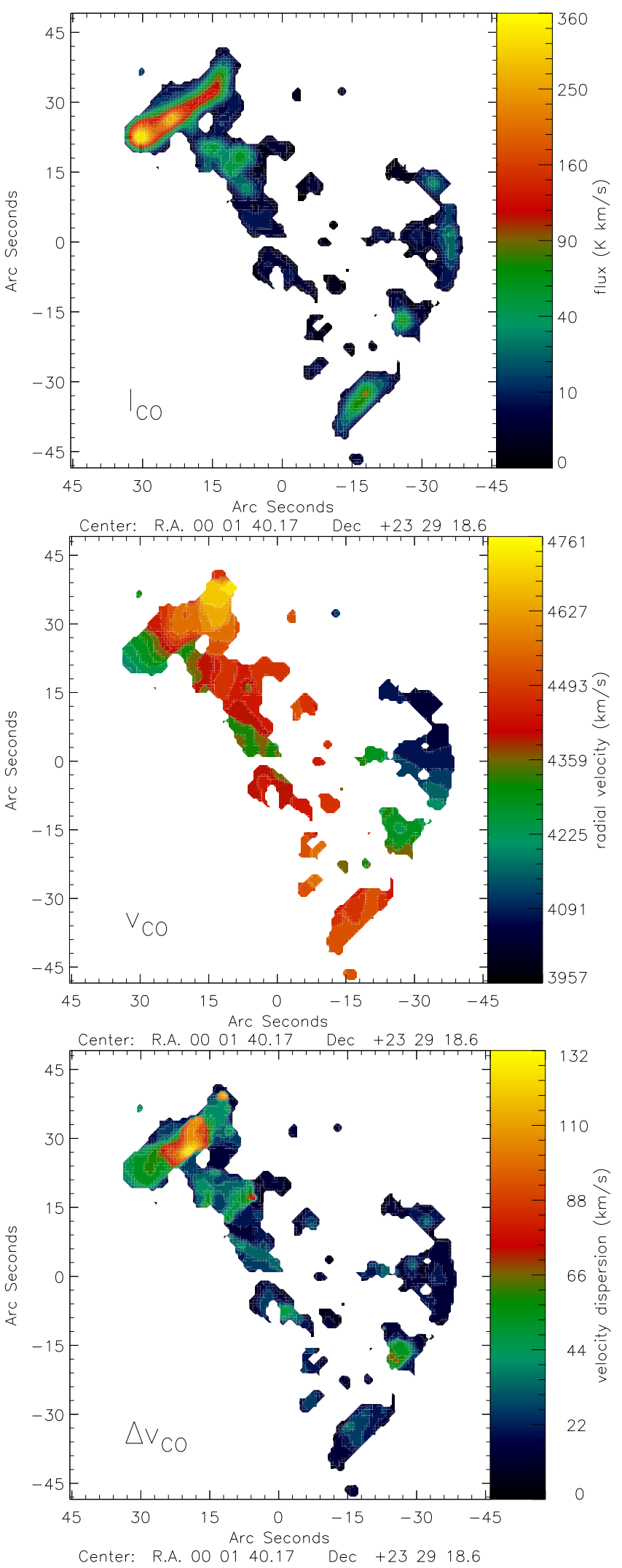

Fig. A.1. Classical $\mathrm{CO}(1-0)$ moment maps.

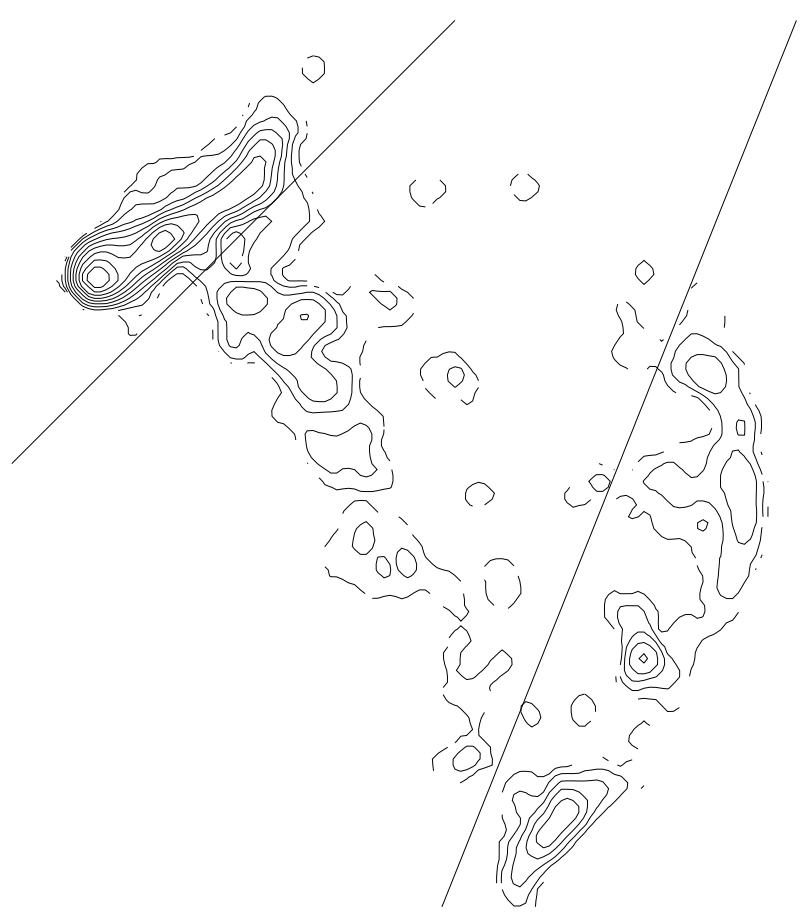

Fig. A.2. Separation between the disk and bridge regions. 
B. Vollmer et al.: Low star formation efficiency due to turbulent adiabatic compression in the Taffy bridge

Table A.1. Molecular entities from CPROPS.

\begin{tabular}{|c|c|c|c|c|c|c|c|}
\hline Number & $\begin{array}{c}\text { Total number } \\
\text { of pixels }\end{array}$ & $\begin{array}{c}\mathrm{RA} \\
\text { (degrees) } \\
\end{array}$ & $\begin{array}{c}\text { Dec } \\
\text { (degrees) } \\
\end{array}$ & $\begin{array}{c}\operatorname{Radius}^{(a)} \\
(\mathrm{pc})\end{array}$ & $\begin{array}{c}\text { Velocity dispersion } \\
\left(\mathrm{km} \mathrm{s}^{-1}\right)\end{array}$ & $\begin{array}{c}\text { Flux } \\
\left(\mathrm{Kkm} \mathrm{s}^{-1} \mathrm{pc}^{2}\right) \\
\end{array}$ & Region \\
\hline 1 & 545 & 0.42114 & 23.4988 & 511.47 & 14.18 & $4.29 E+07$ & UGC 12915 \\
\hline 2 & 1011 & 0.42180 & 23.4981 & 568.03 & 16.88 & $1.02 E+08$ & UGC 12915 \\
\hline 3 & 1425 & 0.42218 & 23.4973 & 1011.25 & 15.93 & $1.62 E+08$ & UGC 12915 \\
\hline 4 & 2297 & 0.42189 & 23.4975 & 1023.43 & 31.56 & $1.89 E+08$ & UGC 12915 \\
\hline 5 & 2558 & 0.42401 & 23.4964 & 772.34 & 44.94 & $2.43 E+08$ & UGC 12915 \\
\hline 6 & 1271 & 0.42102 & 23.4959 & 928.04 & 21.25 & $8.21 E+07$ & bridge \\
\hline 7 & 238 & 0.41837 & 23.4943 & $<300$ & 12.99 & $1.28 E+07$ & bridge \\
\hline 8 & 1117 & 0.42283 & 23.4968 & 647.71 & 29.10 & $8.99 E+07$ & UGC 12915 \\
\hline 9 & 2228 & 0.42013 & 23.4936 & 764.19 & 50.21 & $1.66 E+08$ & bridge \\
\hline 10 & 1361 & 0.42100 & 23.4929 & 634.85 & 30.11 & $1.06 E+08$ & bridge \\
\hline 11 & 465 & 0.41774 & 23.4939 & 728.04 & 14.64 & $2.55 E+07$ & bridge \\
\hline 12 & 108 & 0.41811 & 23.4906 & $<300$ & 18.04 & $6.71 E+06$ & bridge \\
\hline 13 & 1994 & 0.42213 & 23.4939 & 952.07 & 30.94 & $1.51 E+08$ & bridge \\
\hline 14 & 251 & 0.41844 & 23.4903 & 415.32 & 15.90 & $1.39 E+07$ & bridge \\
\hline 15 & 1789 & 0.41984 & 23.4917 & 970.35 & 23.51 & $1.29 E+08$ & bridge \\
\hline 16 & 4934 & 0.42521 & 23.4956 & 1117.62 & 65.40 & $6.44 E+08$ & UGC 12915 \\
\hline 17 & 266 & 0.41870 & 23.4900 & 664.70 & 11.04 & $1.50 E+07$ & bridge \\
\hline 18 & 1710 & 0.41928 & 23.4896 & 1126.03 & 38.68 & $8.90 E+07$ & bridge \\
\hline 19 & 241 & 0.41960 & 23.4931 & 540.82 & 19.70 & $1.46 E+07$ & bridge \\
\hline 20 & 703 & 0.42252 & 23.4937 & 803.97 & 19.81 & $3.74 E+07$ & bridge \\
\hline 21 & 2203 & 0.42356 & 23.4960 & 1167.63 & 44.71 & $1.77 E+08$ & UGC 12915 \\
\hline 22 & 91 & 0.42224 & 23.4925 & $<300$ & 24.26 & $6.46 E+06$ & bridge \\
\hline 23 & 4616 & 0.42654 & 23.4948 & 826.16 & 50.50 & $7.31 E+08$ & UGC 12915 \\
\hline 24 & 216 & 0.41495 & 23.4819 & $<300$ & 10.53 & $1.02 E+07$ & bridge \\
\hline 25 & 173 & 0.41409 & 23.4835 & 547.85 & 7.18 & $1.01 E+07$ & bridge \\
\hline 26 & 61 & 0.41532 & 23.4828 & 81.03 & 7.20 & $2.78 E+06$ & bridge \\
\hline 27 & 100 & 0.41535 & 23.4831 & 747.03 & 7.08 & $7.17 E+06$ & bridge \\
\hline 28 & 290 & 0.41527 & 23.4808 & $<300$ & 26.86 & $1.79 E+07$ & bridge \\
\hline 29 & 2111 & 0.41181 & 23.4795 & 954.00 & 32.61 & $2.17 E+08$ & UGC 12914 \\
\hline 30 & 266 & 0.41543 & 23.4809 & 649.93 & 18.41 & $1.76 E+07$ & bridge \\
\hline 31 & 187 & 0.41557 & 23.4838 & $<300$ & 16.24 & $1.08 E+07$ & bridge \\
\hline 32 & 1744 & 0.41296 & 23.4783 & 996.61 & 33.99 & $2.03 E+08$ & UGC 12914 \\
\hline 33 & 329 & 0.41342 & 23.4797 & 593.83 & 16.89 & $2.41 E+07$ & UGC 12914 \\
\hline 34 & 80 & 0.40977 & 23.4816 & $<300$ & 9.99 & $5.00 E+06$ & UGC 12914 \\
\hline 35 & 95 & 0.41991 & 23.5007 & $<300$ & 11.19 & $6.79 E+06$ & UGC 12915 \\
\hline 36 & 330 & 0.41602 & 23.4918 & 507.34 & 13.51 & $1.78 E+07$ & bridge \\
\hline 37 & 196 & 0.41553 & 23.4918 & 506.89 & 10.65 & $1.14 E+07$ & bridge \\
\hline 38 & 158 & 0.41528 & 23.4917 & $<300$ & 10.93 & $8.12 E+06$ & bridge \\
\hline 39 & 178 & 0.41645 & 23.4972 & $<300$ & 16.71 & $1.12 E+07$ & bridge \\
\hline 40 & 354 & 0.41408 & 23.4860 & 681.41 & 16.46 & $1.67 E+07$ & bridge \\
\hline 41 & 90 & 0.41808 & 23.4858 & $<300$ & 14.51 & $5.77 E+06$ & bridge \\
\hline 42 & 134 & 0.41828 & 23.4870 & $<300$ & 14.96 & $8.86 E+06$ & bridge \\
\hline 43 & 115 & 0.41652 & 23.4860 & $<300$ & 10.12 & $7.26 E+06$ & bridge \\
\hline 44 & 387 & 0.41592 & 23.4857 & 751.19 & 13.80 & $2.04 E+07$ & bridge \\
\hline 45 & 177 & 0.41833 & 23.4861 & 517.13 & 14.99 & $1.10 E+07$ & bridge \\
\hline 46 & 522 & 0.41873 & 23.4870 & 933.92 & 23.21 & $3.01 E+07$ & bridge \\
\hline 47 & 167 & 0.41854 & 23.4877 & 612.88 & 7.06 & $8.79 E+06$ & bridge \\
\hline 48 & 558 & 0.41720 & 23.4867 & 659.95 & 17.79 & $3.10 E+07$ & bridge \\
\hline 49 & 241 & 0.42128 & 23.4994 & $<300$ & 14.52 & $2.13 E+07$ & UGC 12915 \\
\hline 50 & 208 & 0.41488 & 23.4885 & 667.54 & 27.73 & $1.06 E+07$ & bridge \\
\hline 51 & 276 & 0.40974 & 23.4832 & 639.13 & 17.27 & $2.40 E+07$ & UGC 12914 \\
\hline 52 & 1519 & 0.40962 & 23.4839 & 1117.39 & 27.38 & $1.07 E+08$ & UGC 12914 \\
\hline 53 & 159 & 0.40844 & 23.4840 & $<300$ & 14.64 & $1.34 E+07$ & UGC 12914 \\
\hline 54 & 621 & 0.40938 & 23.4841 & 1006.50 & 13.26 & $4.44 E+07$ & UGC 12914 \\
\hline 55 & 46 & 0.40739 & 23.4846 & $<300$ & 10.67 & $4.97 E+06$ & UGC 12914 \\
\hline 56 & 270 & 0.40965 & 23.4839 & 296.58 & 10.49 & $1.73 E+07$ & UGC 12914 \\
\hline 57 & 57 & 0.40968 & 23.4887 & $<300$ & 17.80 & $4.59 E+06$ & UGC 12914 \\
\hline 58 & 202 & 0.40989 & 23.4845 & $<300$ & 14.72 & $1.33 E+07$ & UGC 12914 \\
\hline 59 & 118 & 0.41046 & 23.4875 & 348.53 & 26.59 & $8.11 E+06$ & UGC 12914 \\
\hline 60 & 144 & 0.40863 & 23.4875 & 300.83 & 12.13 & $9.22 E+06$ & UGC 12914 \\
\hline
\end{tabular}


Table A.1. continued.

\begin{tabular}{lccccccc}
\hline \hline Number & $\begin{array}{c}\text { Total number } \\
\text { of pixels }\end{array}$ & $\begin{array}{c}\text { RA } \\
(\text { degrees })\end{array}$ & $\begin{array}{c}\text { Dec } \\
(\text { degrees })\end{array}$ & $\begin{array}{c}\text { Radius } \\
(\mathrm{pc})\end{array}$ & $\begin{array}{c}\text { Velocity dispersion } \\
\left(\mathrm{km} \mathrm{s}^{-1}\right)\end{array}$ & $\begin{array}{c}\text { Flux } \\
\left(\mathrm{K} \mathrm{km} \mathrm{s}^{-1} \mathrm{pc}^{2}\right)\end{array}$ & Region \\
\hline 61 & 90 & 0.40882 & 23.4890 & 160.47 & 11.36 & $6.39 E+06$ & UGC 12914 \\
62 & 146 & 0.40985 & 23.4885 & $<300$ & 18.75 & $7.45 E+06$ & UGC 12914 \\
63 & 58 & 0.40761 & 23.4918 & $<300$ & 6.85 & $3.86 E+06$ & UGC 12914 \\
64 & 1585 & 0.40674 & 23.4871 & 1051.63 & 42.77 & $1.29 E+08$ & UGC 12914 \\
65 & 72 & 0.40762 & 23.4868 & $<300$ & 9.30 & $5.36 E+06$ & UGC 12914 \\
66 & 506 & 0.40906 & 23.4888 & 771.13 & 19.15 & $2.91 E+07$ & UGC 12914 \\
67 & 341 & 0.40788 & 23.4920 & 577.13 & 11.67 & $2.34 E+07$ & UGC 12914 \\
68 & 550 & 0.40814 & 23.4888 & 765.39 & 13.50 & $3.29 E+07$ & UGC 12914 \\
69 & 1118 & 0.40682 & 23.4891 & 873.53 & 16.26 & $1.05 E+08$ & UGC 12914 \\
70 & 465 & 0.40992 & 23.4927 & 1181.87 & 19.69 & $2.81 E+07$ & bridge \\
71 & 597 & 0.40682 & 23.4908 & 503.07 & 14.06 & $5.44 E+07$ & UGC 12914 \\
72 & 1130 & 0.40775 & 23.4924 & 1028.35 & 18.64 & $9.86 E+07$ & UGC 12914 \\
73 & 85 & 0.41281 & 23.4818 & 619.77 & 12.53 & $8.16 E+06$ & UGC 12914 \\
74 & 72 & 0.41328 & 23.4824 & $<300$ & 20.01 & $4.29 E+06$ & UGC 12914 \\
75 & 82 & 0.42309 & 23.4982 & $<300$ & 11.65 & $7.60 E+06$ & UGC 12915 \\
76 & 79 & 0.41153 & 23.4822 & $<300$ & 6.95 & $4.81 E+06$ & UGC 12914 \\
77 & 82 & 0.41154 & 23.4824 & $<300$ & 11.28 & $5.93 E+06$ & UGC 12914 \\
78 & 477 & 0.41114 & 23.4887 & $<300$ & 29.15 & $2.68 E+07$ & bridge \\
79 & 120 & 0.41332 & 23.4973 & 224.35 & 11.56 & $9.96 E+06$ & bridge \\
80 & 65 & 0.40959 & 23.4948 & $<300$ & 7.42 & $4.48 E+06$ & bridge \\
\hline
\end{tabular}

Notes. ${ }^{(a)}$ When CPROPS was unable to deconvolve the cloud size, we put $<300$ pc.

\section{Appendix B: Additional moment maps and 3D views of the datacubes}
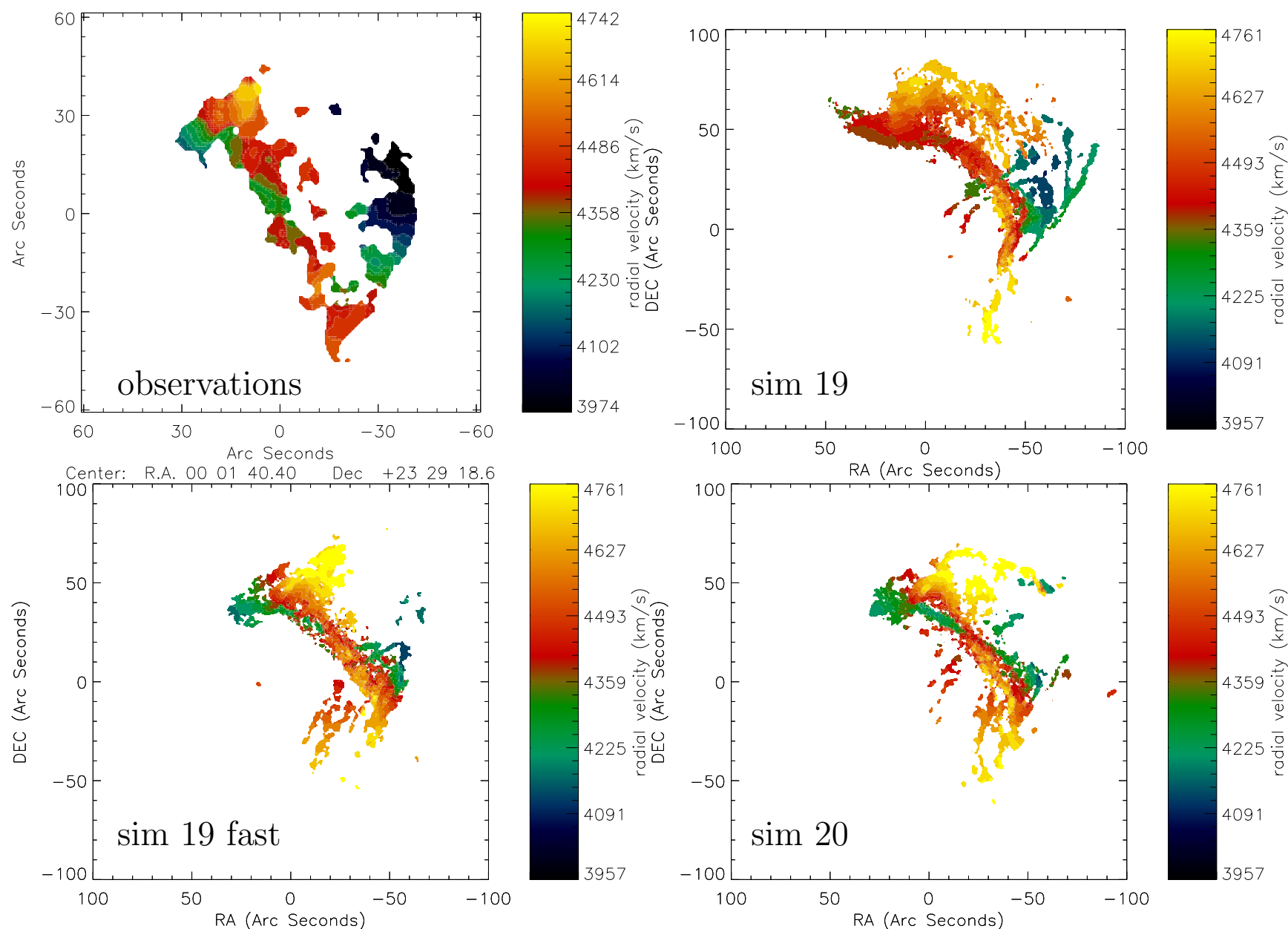

Fig. B.1. $\mathrm{CO}(1-0)$ moment 1 maps together with the model $\mathrm{H}_{2}$ moment 0 maps. Upper left panel: PdBI observations. Other panels: simulations. 
B. Vollmer et al.: Low star formation efficiency due to turbulent adiabatic compression in the Taffy bridge
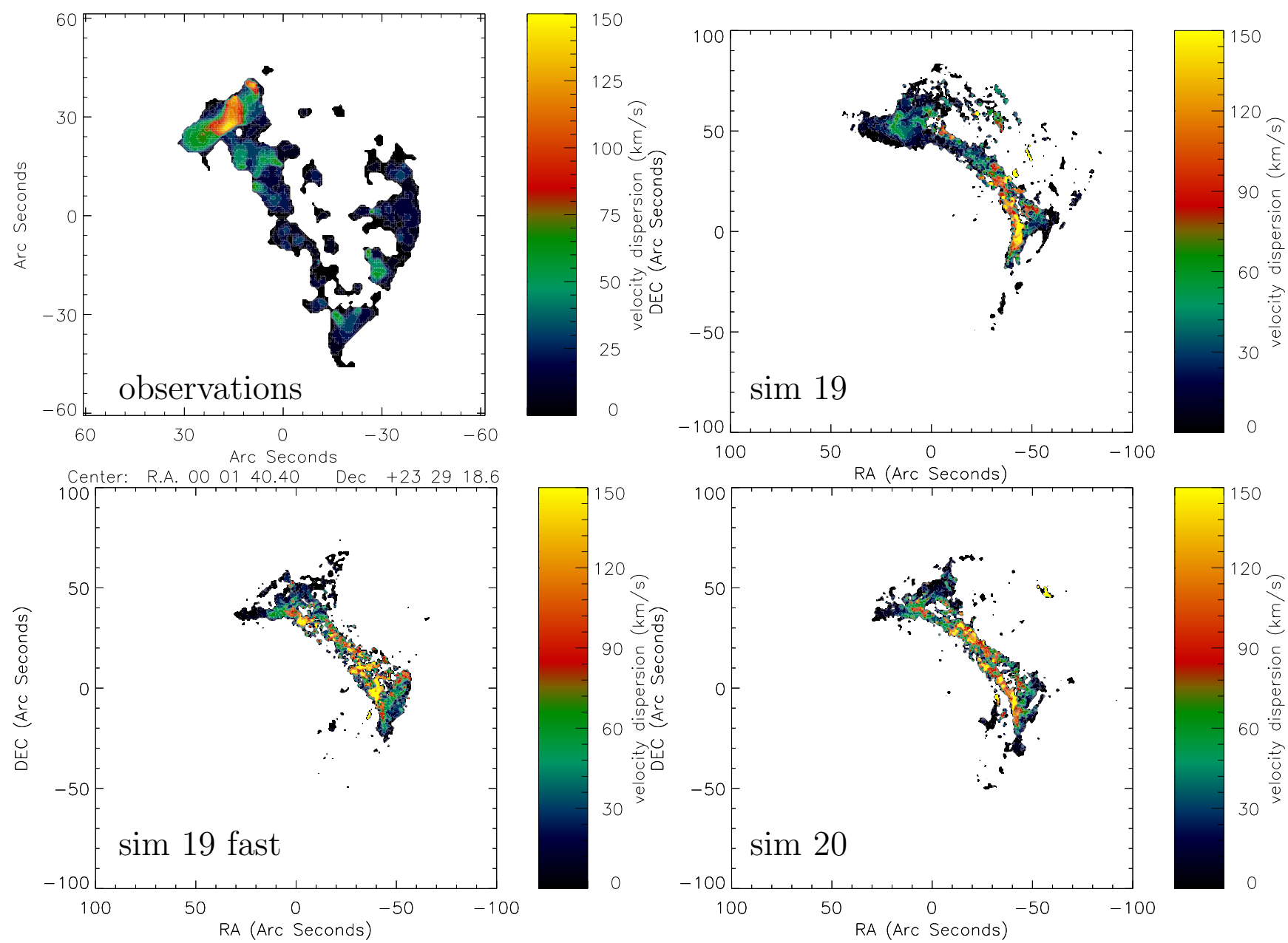

Fig. B.2. $\mathrm{CO}(1-0)$ moment 2 maps together with the model $\mathrm{H}_{2}$ moment 0 maps. Upper left panel: PdBI observations. Other panels: simulations. 

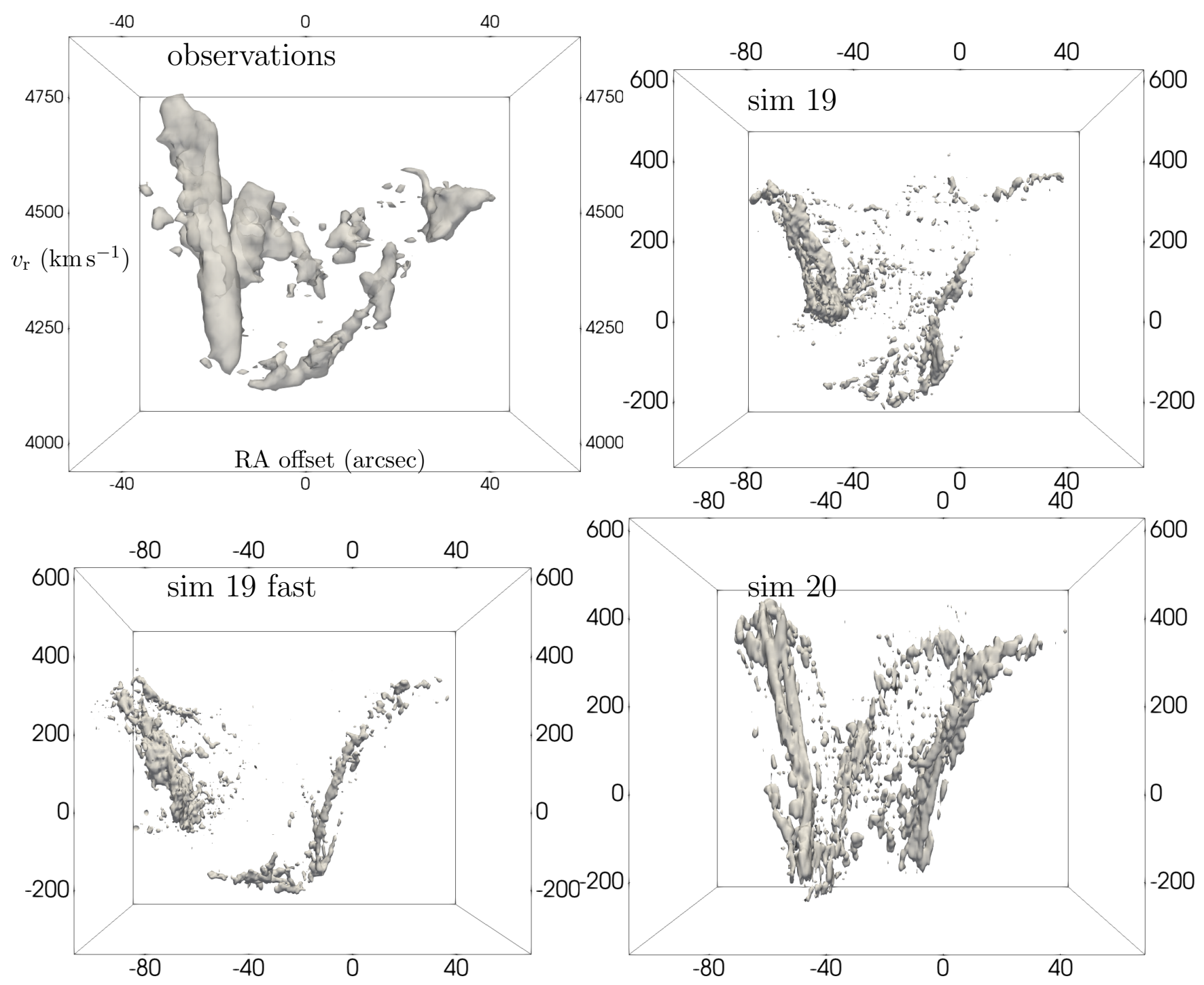

Fig. B.3. Second $3 \mathrm{D}$ view of the observed $\mathrm{CO}(1-0)$ datacube and the model $\mathrm{H}_{2}$ datacubes. The axis labels are only shown for the observations. For a better understanding of these views, three 3D animations of the rotating datacube are available online (taffy_cube3D_z.gif, taffy_cube3D_z1.gif, and taffy_cube3D_x.gif). 
B. Vollmer et al.: Low star formation efficiency due to turbulent adiabatic compression in the Taffy bridge

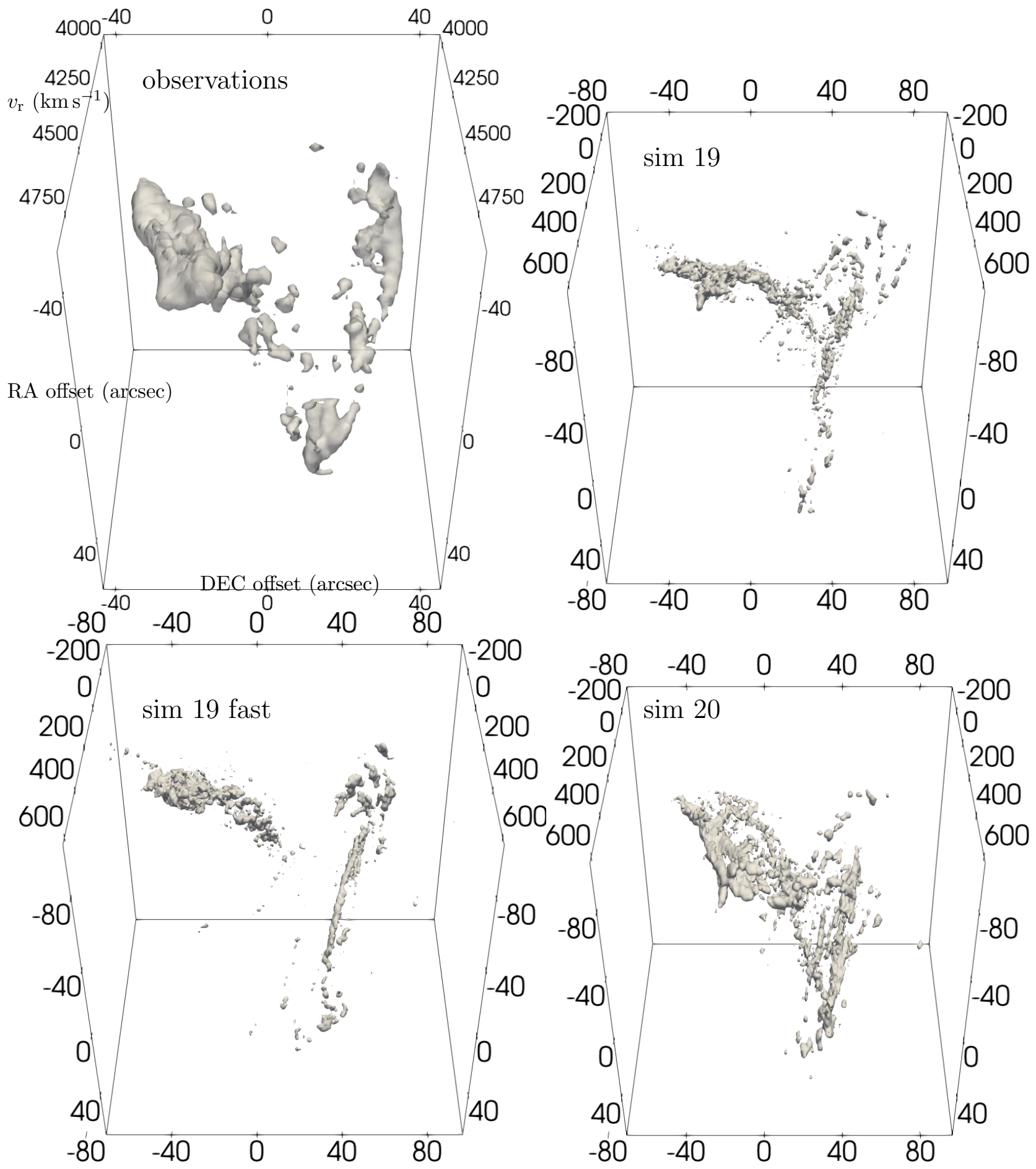

Fig. B.4. Third 3D view of the observed $\mathrm{CO}(1-0)$ datacube and the model $\mathrm{H}_{2}$ datacubes. The axis labels are only shown for the observations. For a better understanding of these views, three 3D animations of the rotating datacube are available online (taffy_cube3D_z.gif, taffy_cube3D_z1.gif, and taffy_cube3D_x.gif). 
A\&A 647, A138 (2021)
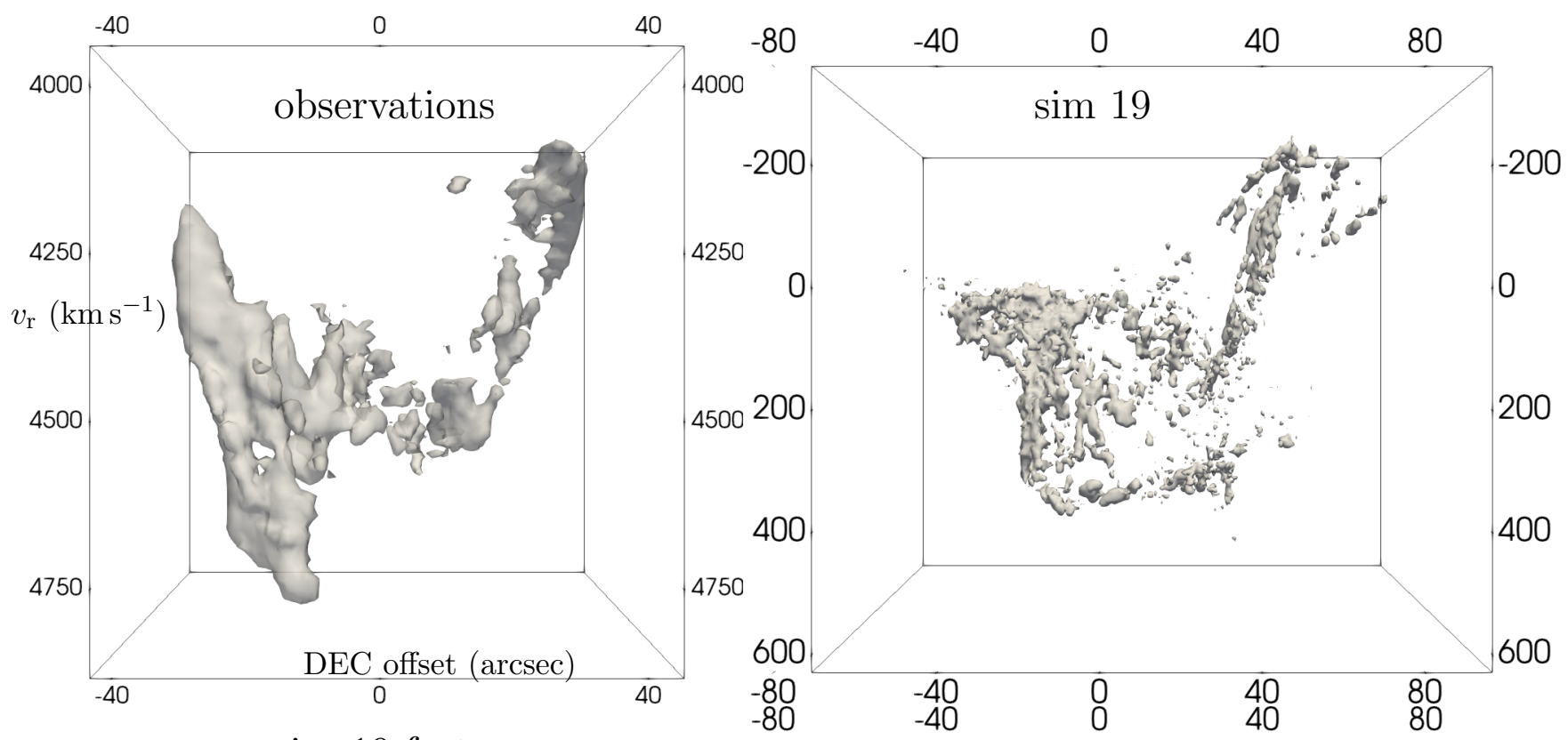

sim 19 fast
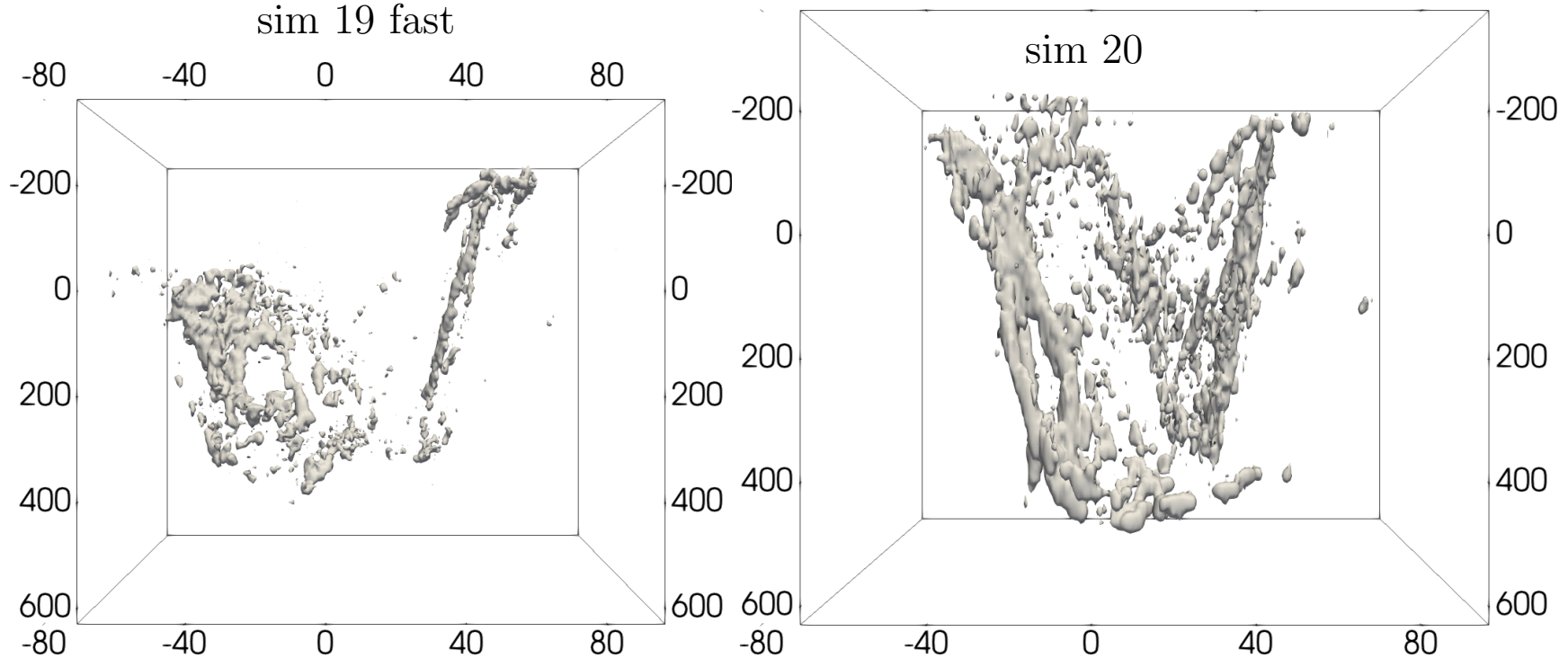

Fig. B.5. Fourth 3D view of the observed $\mathrm{CO}(1-0)$ datacube and the model $\mathrm{H}_{2}$ datacubes. The axis labels are only shown for the observations. For a better understanding of these views, three 3D animations of the rotating datacube are available online (taffy_cube3D_z.gif, taffy_cube3D_z1.gif, and taffy_cube3D_x.gif). 


\section{Appendix C: Comparison with models}

Our modeling effort is based on the combination of a large-scale dynamical model (Appendix C.1) together with a small-scale analytical model (Appendix C.3) to handle the properties of a turbulent ISM in a simplified way. All cloud-cloud collisions conserve mass and momentum. Our method is akin to a stickyparticle scheme (for example Combes \& Gerin 1985) where the cloud-cloud collisions are resolved due to the high time resolution. The dynamical simulations follow Boltzmann's equation with a collisional term involving binary partially inelastic collisions.

The simulations do not include stellar feedback and do not follow the thermal evolution of the gas. For the thermal evolution of the gas in a galaxy-galaxy head-on collision we rely on the results of Yeager \& Struck (2019). Our star formation recipe is based on cloud-cloud collisions (Appendix C.2). We verified that our SFR recipe based on cloud-cloud collisions leads to Schmidt-like star formation law $\dot{\rho}_{*} \propto \rho^{1.5}$.

Following Robertson \& Goldreich (2012) and Mandal et al. (2020), we expect turbulent adiabatic heating to occur when the gas compression is faster than dissipation of turbulence $t_{\text {diss }}$ (Appendix C.4). Since $t_{\text {diss }}$ is not available from the dynamical model, we compare $t_{\text {comp }}$ to the $t_{\text {diss }}$ the gas would have if it formed stars as in a galactic disk (in other words following a Kennicutt-Schmidt law). When compression energy exceeds that of stellar feedback, the velocity dispersion is expected to increase. In this case, we assume that the velocity dispersion of the clouds also increases such that star formation will be significantly reduced (Appendix C.5).

\section{C.1. Large-scale dynamics - The dynamical model}

We used the dynamical simulations of Vollmer et al. (2012a). The ISM is simulated as a collisional component, that is as discrete particles that possess a mass and a radius and can have partially inelastic collisions. In contrast to smoothed particle hydrodynamics (SPH), which is a quasi-continuous approach where the particles cannot penetrate each other, our approach allows a finite penetration length, which is given by the massradius relation of the particles. During the disk evolution, the cloud particles can have partially inelastic collisions, the outcome of which (coalescence, mass exchange, or fragmentation) is simplified following the geometrical prescriptions of Wiegel (1994).

The particle trajectories are integrated using an adaptive timestep for each particle. This method is described in Springel et al. (2001). The criterion for an individual timestep is $\Delta t_{i}=$ $5 \mathrm{~km} \mathrm{~s}^{-1} / a_{i}$, where $a_{i}$ is the acceleration of the particle $i$. The minimum value of $\Delta t_{i}$ defines the global timestep used for the Burlisch-Stoer integrator that integrates the collisional component. The global timestep ${ }^{4}$ is typically around $10^{4} \mathrm{yr}$. For a velocity of $1000 \mathrm{~km} \mathrm{~s}^{-1}$ this corresponds to $\sim 10 \mathrm{pc}$.

During each cloud-cloud collision the overlapping parts of the clouds are calculated. Let $b$ be impact parameter and $r_{1}$ and $r_{2}$ the radii of the larger and smaller clouds. If $r_{1}+r_{2}>b>$ $r_{1}-r_{2}$ the collision can result into fragmentation (high-speed encounter) or mass exchange. If $b<r_{1}-r_{2}$ mass exchange or coalescence (low-speed encounter) can occur. If the maximum number of gas particles or cloud (40000) is reached, only coalescent or mass exchanging collisions are allowed. In this way a cloud mass distribution is naturally produced. The cloud

\footnotetext{
4 In addition, the integrator divided this timestep into at least three subtimesteps of about $3000 \mathrm{yr}$.
}

masses and velocities resulting from a cloud-cloud collision are calculated by assuming mass and momentum conservation. In Vollmer et al. (2012a) we normalized the mass-size relation of the model clouds such that the gas mass of the bridge agrees with that derived from $\mathrm{CO}$ observations of the Taffy system. The cloud particle masses and radii range between $10^{4}$ and $10^{6} M_{\odot}$ and 35 and $145 \mathrm{pc}$, respectively. The gas particles/clouds cannot be taken as the real clouds in the ISM of galactic disks, because the lifetime of giant molecular clouds (GMCs) of several $10 \mathrm{Myr}$ (e.g., Zamora-Avilés \& Vázquez-Semadeni 2014) does not permit frequent GMC-GMC collisions. On the other hand, during an ISM-ISM collision as in the Taffy system, there will be a significant number of GMC-GMC collisions since the collision time is small $t \sim 1 \mathrm{kpc} /\left(1000 \mathrm{~km} \mathrm{~s}^{-1}\right)=1 \mathrm{Myr}$. Following the direct cloud-cloud collision scenario of Harwit et al. (1987), the gas is heated to temperatures corresponding to a sizable fraction of the kinetic energy of the collision (millions of K). The shock-heated gas will then cool down with a rate that depends on its density. For a density of $10^{3} \mathrm{~cm}^{-3}$ the cooling rate is about $10^{4} \mathrm{yr}$ (Harwit et al. 1987). It should be noted that there will also be collisions between the clouds and more diffuse gas as simulated by Yeager \& Struck (2020). Since we are only interested in the dense molecular gas, our cloud particles can be identified with cool gas a few megayears after impact.

\section{C.2. Star formation}

In numerical simulations, the star formation recipe usually involves the gas density $\rho$ and the free-fall time $t_{\mathrm{ff}}=$ $\sqrt{3 \pi /(32 G \rho)}: \dot{\rho}_{*} \propto \rho t_{\mathrm{ff}}^{-1} \propto \rho^{1.5}$. In our dynamical model the SFR is proportional to the cloud-cloud collision rate and stars are formed in cloud-cloud collisions.

The newly created star particles have zero mass (they are test particles) and the positions and velocities of the colliding clouds after the collision. These particles then move passively with the whole system. The information about the time of creation is attached to each newly created star particle. The UV emission of a star particle in the two GALEX bands is modeled by the UV flux from single stellar population models from STARBURST99 (Leitherer et al. 1999). The age of the stellar population equals the time since the creation of the star particle. The total UV distribution is then the extinction-free distribution of the UV emission of the newly created star particles.

We verified that our SFR recipe based on cloud-cloud collisions leads to the same exponent (1.4-1.6; Fig. C.1) of the gas density in a simulation of an isolated spiral galaxy and for the Taffy system at impact and $\sim 20 \mathrm{Myr}$ after impact. As a consequence, our code reproduces the observed SFR-total gas surface density, SFR-molecular gas surface density, and SFR-stellar surface density relations (Vollmer et al. 2012b). To go a step further we show the comparison of our model results with observed scaling relations for the molecular gas surface density, star formation rate, and star formation efficiency in Fig. C.2. The model relations agree quite well with the observed relations.

\section{C.3. Small-scale ISM properties - The analytical model}

The model of Vollmer \& Beckert (2003) and Vollmer \& Leroy (2011) considers the warm, cold, and molecular phases of the ISM as a single turbulent gas. The gas is taken to be clumpy, so that the local density can be enhanced relative to the average density of the disk. From the local density, the free-fall time of an individual self-gravitating gas clump is used as the timescale 

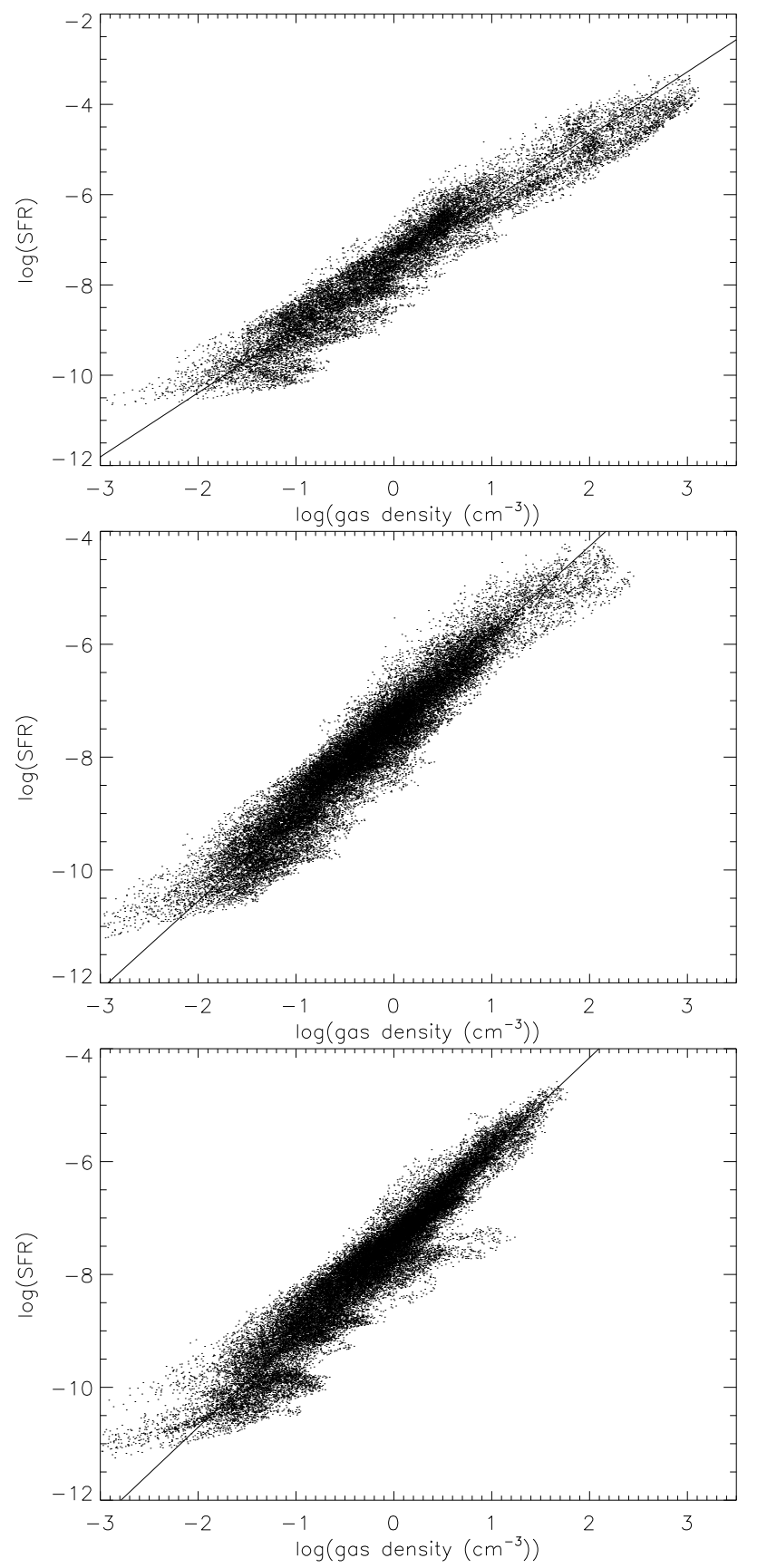

Fig. C.1. Local SFR $\dot{\rho}_{*}$ (in arbitrary units) as a function of the volume density $\rho$. Upper panel: unperturbed simulation after $0.5 \mathrm{Gyr}$. Middle panel: simulation 19 at impact. Lower panel: simulation $1920 \mathrm{Myr}$ after impact. Solid lines show linear regressions. The slope of the correlation $\dot{\rho}_{*} \propto \rho^{n}$ for the unperturbed galaxy simulation is $n=1.4$, whereas it is $n=1.6$ for the Taffy simulation.

governing star formation. The SFR is used to calculate the rate of energy injection by supernovae. Turbulence is driven by this energy injection into turbulent eddies that have a characteristic length scale $l_{\text {driv }}$ and a characteristic velocity $v_{\text {turb }} ; l_{\text {driv }}$ and $v_{\text {turb }}$ are linked to the volume filling factor of self-gravitating GMCs $\Phi_{\mathrm{V}}$. All model parameters are described in Table C.1. The Vollmer \& Beckert (2003) model does not address the spatial inhomogeneity of the turbulent driving nor the mechanics of turbulent driving and dissipation. It is assumed that the energy input rate into the ISM due to supernovae is cascaded to smaller scales without loss. The energy of self-gravitating clouds is dis-
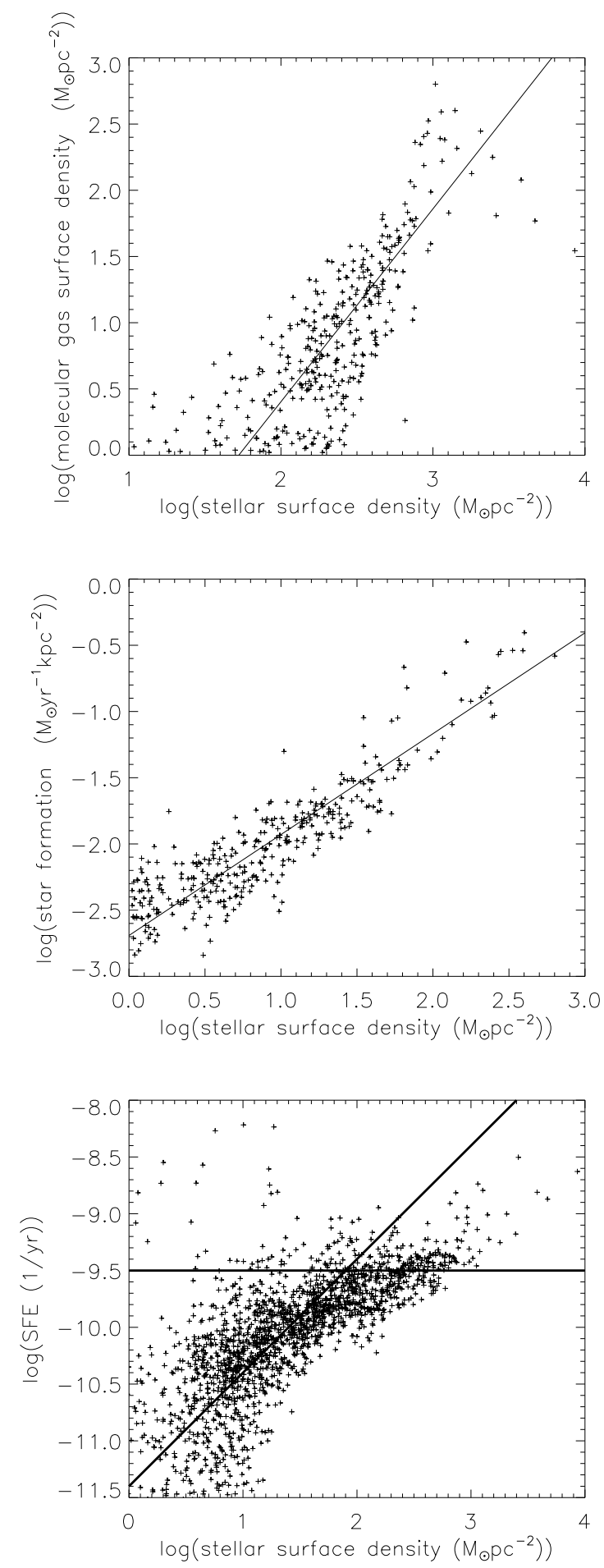

Fig. C.2. Model of an unperturbed spiral galaxy. Upper panel: SFR as a function of the molecular gas surface density. Middle panel: SFR as a function of the stellar surface density. Lower panel: star formation efficiency with respect to the molecular gas as a function of the stellar surface density. The solid lines mark the observed relations found by Leroy et al. (2008).

sipated via cloud contraction and star formation. The smallest scale investigated by the analytical model is the scale where the gas clouds become self-gravitating. The size, density, and turbulent crossing time of these clouds are $l_{\mathrm{cl}}=l_{\text {driv }} / \delta, \rho_{\mathrm{cl}}=\langle\rho\rangle / \Phi_{\mathrm{V}}$, and $t_{\text {turb,cl }}=l_{\text {cl }} / v_{\text {turb,cl }}=\delta^{-0.5} l_{\text {driv }} / v_{\text {turb }}$, where $\langle\rho\rangle$ is the largescale gas density. 
Table C.1. Model parameters.

\begin{tabular}{|c|c|c|}
\hline Parameter & Unit & Explanation \\
\hline$G=5 \times 10^{-15}$ & $\mathrm{pc}^{3} \mathrm{yr}^{-1} M_{\odot}^{-1}$ & Gravitation constant \\
\hline$l_{\text {driv }}$ & $\mathrm{pc}$ & Turbulent driving length scale \\
\hline$v_{\text {turb }}$ & $\mathrm{pc} \mathrm{yr}^{-1}$ & Gas turbulent 3D velocity dispersion at $l_{\text {driv }}$ \\
\hline$l_{\mathrm{cl}}$ & $\mathrm{pc}$ & Cloud size \\
\hline$v_{\text {turb,cl }}$ & $\mathrm{pc} \mathrm{yr}^{-1}$ & Cloud 3D velocity dispersion \\
\hline$\sigma_{\mathrm{cl}}$ & $\mathrm{pc} \mathrm{yr}^{-1}$ & Cloud $1 \mathrm{D}$ velocity dispersion \\
\hline$\delta=l_{\text {driv }} / l_{\mathrm{cl}}$ & & Scaling between driving length scale and cloud size \\
\hline$\Phi_{\mathrm{V}}$ & & Volume filling factor of self-gravitating clouds \\
\hline$\langle\rho\rangle$ & $M_{\odot} \mathrm{pc}^{-3}$ & Mean gas density \\
\hline$t_{\mathrm{ff}}=\sqrt{3 \pi /(32 G\langle\rho\rangle)}$ & $\mathrm{yr}$ & \\
\hline$\rho_{\mathrm{cl}}=\langle\rho\rangle / \Phi_{\mathrm{V}}$ & $M_{\odot} \mathrm{pc}^{-3}$ & Cloud density \\
\hline$t_{\mathrm{ff}, \mathrm{cl}}$ & yr & Cloud free fall timescale at size $l_{\mathrm{cl}}$ \\
\hline$t_{\text {turb,cl }}$ & $\mathrm{yr}$ & Cloud turbulent timescale at size $l_{\mathrm{cl}}$ \\
\hline$t_{\text {life,cl }}$ & $\mathrm{yr}$ & Cloud lifetime \\
\hline$t_{\mathrm{dep}}$ & yr & Gas depletion timescale \\
\hline$\dot{\rho}_{*}$ & $M_{\odot} \mathrm{pc}^{-3} \mathrm{yr}^{-1}$ & SFR per unit volume \\
\hline$\xi=4.6 \times 10^{-8}$ & $\mathrm{pc}^{2} \mathrm{yr}^{-2}$ & Constant relating SN energy input to SF \\
\hline$\epsilon_{\mathrm{ff}}$ & & Star formation efficiency per free fall time \\
\hline$f_{\mathrm{SF}}$ & & Fraction of the star-forming molecular gas mass \\
\hline$\epsilon_{*}$ & & Cloud mass fraction converted into stars \\
\hline$\epsilon_{\text {life }}=t_{\mathrm{ff}, \mathrm{cl}} / t_{\mathrm{life}, \mathrm{cl}}$ & & Cloud free-fall time divided by the lifetime \\
\hline$t_{\text {diss }}=l_{\text {driv }} / v_{\text {turb }}$ & $\mathrm{yr}$ & Turbulent dissipation timescale \\
\hline$t_{\mathrm{comp}}=\rho /(d \rho / d t)$ & $\mathrm{yr}$ & Gas compression timescale \\
\hline
\end{tabular}

Following Vollmer \& Leroy (2011) the SFR per unit volume is given by

$\dot{\rho}_{*}=\Phi_{\mathrm{V}} \rho t_{\mathrm{ff}, \mathrm{cl}}^{-1}=\sqrt{\Phi_{\mathrm{V}}} \rho t_{\mathrm{ff}}^{-1}=\epsilon_{\mathrm{ff}} \rho t_{\mathrm{ff}}^{-1}$,

where $\Phi_{\mathrm{V}}^{-1}=\rho_{\mathrm{cl}} / \rho$ is the overdensity of self-gravitating clouds, $\rho$ the gas density, $t_{\mathrm{ff}, \mathrm{cl}}$ the free-fall time of a self-gravitating gas cloud, $t_{\mathrm{ff}}=\sqrt{3 \pi /(32 G \rho)}$, and $\epsilon_{\mathrm{ff}}=\sqrt{\Phi_{\mathrm{V}}} \propto t_{\mathrm{turb}} / t_{\mathrm{ff}}$ the star formation efficiency per free-fall time. Vollmer et al. (2017) found that for SFRs comparable to those of nearby spiral galaxies and gas velocity dispersions around $10 \mathrm{~km} \mathrm{~s}^{-1}, \Phi_{\mathrm{V}}$ is about constant and has values of a few times 0.001 , consistent with the findings of Leroy et al. (2017) in M 51. In the following we will show that $\epsilon_{\mathrm{ff}}=\sqrt{\Phi_{\mathrm{V}}} \propto v_{\text {turb }}$, which is consistent with the predictions of feedback-regulated star formation in turbulent, self-gravitating, strongly star-forming galactic gas disks (Ostriker \& Shetty 2011; Faucher-Giguère et al. 2013; however, see Krumholz et al. 2018 for a different point of view).

For self-gravitating clouds with a Virial parameter of unity the turbulent crossing time equals twice the free-fall time:

$2 t_{\mathrm{ff}, \mathrm{cl}}=2 \sqrt{\frac{3 \pi \Phi_{\mathrm{V}}}{32 G\langle\rho\rangle}}=\frac{\sqrt{3} l_{\mathrm{cl}}}{2 v_{\mathrm{turb}, \mathrm{cl}}}$,

where $l_{\mathrm{cl}}$ and $v_{\text {turb,cl }}$ are the size and turbulent 3D velocity dispersion of the cloud. Using Larson's law $\left(l_{\mathrm{cl}} / v_{\text {turb,cl }}=\right.$ $\left.l_{\text {driv }} / v_{\text {turb }} / \sqrt{\delta}\right)$, the SFR per unit volume is

$\dot{\rho}_{*}=\frac{4 \sqrt{\delta}}{\sqrt{3}} \Phi_{\mathrm{V}}\langle\rho\rangle v_{\mathrm{turb}} / l_{\text {driv }}$.

We can connect the energy input into the ISM by SNe directly to the SFR. With the assumption of a constant initial mass function independent of environment one can write

$\frac{1}{2}\langle\rho\rangle \frac{v_{\text {turb }}^{3}}{l_{\text {driv }}}=\xi \dot{\rho}_{*}$.

This leads to the following expression for the volume filling factor:

$\Phi_{\mathrm{V}}=\frac{\sqrt{3} v_{\mathrm{turb}}^{2}}{8 \sqrt{\delta} \xi}$

and the star formation law becomes

$\dot{\rho}_{*}=\sqrt{\frac{\sqrt{3}}{8 \sqrt{\delta} \xi}} v_{\mathrm{turb}} \rho t_{\mathrm{ff}}^{-1}$.

We thus find $\epsilon_{\mathrm{ff}} \propto v_{\text {turb }}$, which is equivalent to Eq. (22) of Ostriker \& Shetty (2011), Eq. (37) of Faucher-Giguère et al. (2013), and Eq. (54) of Krumholz et al. (2018).

Using Eqs. (C.1) and (C.4), the large-scale turbulent crossing time, which equals the turbulent dissipation timescale, is

$t_{\mathrm{turb}}=t_{\mathrm{diss}}=\frac{l_{\text {driv }}}{v_{\text {turb }}}=\frac{v_{\text {turb }}^{2}}{2 \xi \sqrt{\Phi_{\mathrm{V}}}} \sqrt{\frac{3 \pi}{32 G\langle\rho\rangle}}$.

Inserting Eqs. (C.5) into (C.7) leads to the final expression for the turbulent dissipation timescale:

$t_{\text {diss }}=v_{\text {turb }} \sqrt{\frac{6 \pi \sqrt{\delta}}{\sqrt{3} 32 G\langle\rho\rangle \xi}}$.

Alternatively, we can assume a constant $\epsilon_{\mathrm{ff}}$ (Krumholz \& McKee 2005; Krumholz et al. 2012). In this case the equation 
for the energy injection and dissipation becomes

$\frac{1}{2}\langle\rho\rangle \frac{v_{\text {turb }}^{3}}{l_{\text {driv }}}=\xi \epsilon_{\mathrm{ff}}\langle\rho\rangle t_{\mathrm{ff}}^{-1}$

and the dissipation timescale is

$t_{\mathrm{diss}, \epsilon}=\frac{v_{\mathrm{turb}}^{2}}{2 \xi \epsilon_{\mathrm{ff}}} \sqrt{\frac{3 \pi}{32 G\langle\rho\rangle}}$.

This timescale equals $t_{\text {diss }}$ (Eq. (C.7)) for $v_{\text {turb }}=\epsilon_{\mathrm{ff}} \sqrt{\frac{8 \xi \sqrt{\delta}}{\sqrt{3}}}=$ $6.5 \mathrm{~km} \mathrm{~s}^{-1}$. For higher velocity dispersions $t_{\text {diss }, \epsilon}>t_{\text {diss }}$.

Within the framework of Vollmer et al. (2017) the dependence of $\epsilon_{\mathrm{ff}}$ on the turbulent velocity dispersion is $\epsilon_{\mathrm{ff}} \propto \sqrt{v_{\text {turb }}}$ leading to $t_{\text {diss }} \propto v_{\text {turb }}^{1.5}$. For $v_{\text {turb }}>10 \mathrm{~km} \mathrm{~s}^{-1}$, Eq. (C.8) represents the lower limit for the dissipation timescale. Since we require $t_{\text {comp }}<t_{\text {diss }}$ for turbulent adiabatic compression, this lower limit of $t_{\text {diss }}$ is an appropriate, conservative choice.

The dissipation timescale $t_{\text {diss }}$ is compared to the compression timescale $t_{\text {comp }}$ for the quiet disks before the interaction in Fig. C.3 and for the system $\sim 20 \mathrm{Myr}$ after impact in Fig. C.4. The dissipation timescale of the quiet disks (right panel of Fig. C.3) shows the $1 / \sqrt{\langle\rho\rangle}$-dependence of Eq. (C.8). Roughly half of the particles have a $1 \mathrm{D}$ velocity dispersion of about $10 \mathrm{~km} \mathrm{~s}^{-1}$ (green contours), and about $25 \%$ have twice that velocity dispersion. Three quarters of all particles have $t_{\text {comp }}>t_{\text {diss }}$ (left panel of Fig. C.3).

The picture changes for the system at the time of interest where we geometrically divided the system into a bridge and disk+tidal tail regions. The majority of the gas particles of the system show significantly higher velocity dispersions and thus higher $t_{\text {diss }}$ (right panels of Fig. C.4). At the same time the compression timescale of the majority of particles is significantly shorter than those of the quiet disks (left panels of Fig. C.4). About half of the particles have $t_{\text {comp }}<t_{\text {diss. }}$. The gas densities in the bridge do not exceed $\langle\rho\rangle \sim 10 \mathrm{~cm}^{-1}$ (lower panels of Fig. C.4) which is due to the coarse spatial resolution of our simulations. The gas particles located within the bridge region almost exclusively have high velocity dispersions (lower right panel of Fig. C.4) and show $t_{\text {comp }}<t_{\text {diss }}$ (lower left panel of Fig. C.4).

\section{C.4. Turbulent adiabatic compression}

In our simulation of an isolated spiral galaxy the $1 \mathrm{D}$ velocity dispersion of the model clouds is constant, $v_{\text {disp }} \sim 10 \mathrm{~km} \mathrm{~s}^{-1}$, during 1 Gyr. Since there is no stellar feedback, the cloud velocity dispersion is increased when the gas is compressed. In kinetic theory, particles move with random motions around the sound speed and over a length scale given by the collision mean free path. In the eddy-viscosity model (Boussinesq approximation), eddies also move with random motions, at a typical speed given by the turbulent velocity dispersion and over a typical length scale called the mixing length. Since these timescales are well resolved in our simulations, we can identify the particle/cloud velocity dispersion with the velocity dispersion of the largest turbulent eddies.

Since the dissipation timescale is not part of the dynamical model, we compare the gas compression timescale to the turbulent dissipation timescale $t_{\text {diss }}=l_{\text {driv }} / v_{\text {turb }}$ following Eq. (C.8), meaning in the absence of adiabatic compression (Eq. C.4). The large-scale velocity dispersion and density are taken from the
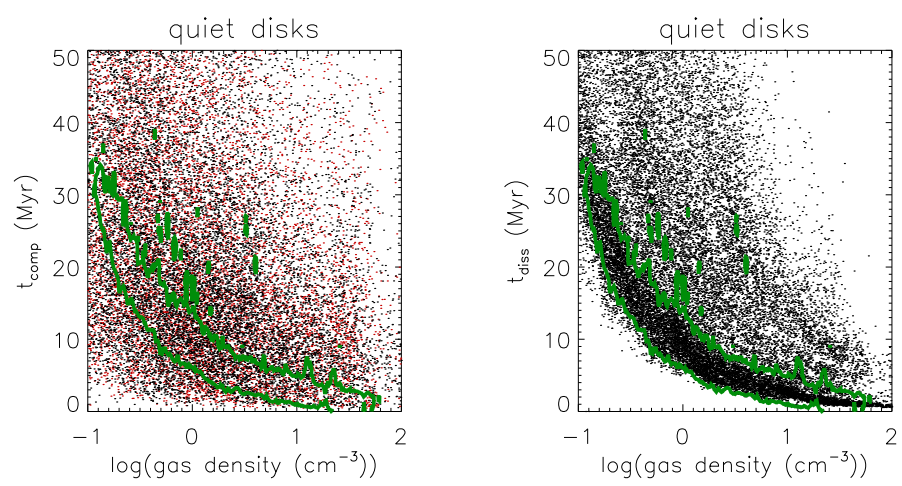

Fig. C.3. Compression (Eq. (C.8), left panel) and dissipation (Eq. (C.8), right panel) timescales as a function of the mean gas density $\langle\rho\rangle$ for the quite disks before the interaction. Negative compression timescales, namely gas expansion, are marked as red points in the left panel. The green contours mark the regions of highest particle density in the $\langle\rho\rangle-$ $t_{\text {diss }}$ relation.
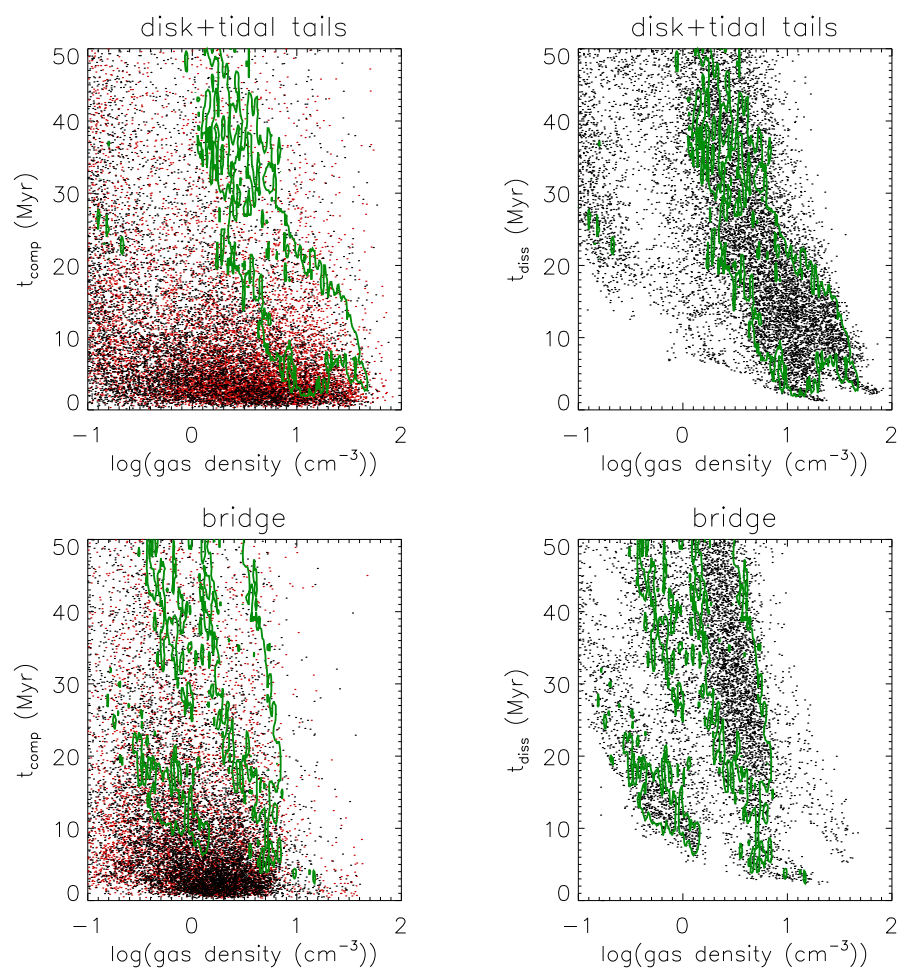

Fig. C.4. Compression (Eq. (C.8), left panel) and dissipation (Eq. (C.8), right panel) timescales as a function of the mean gas density $\langle\rho\rangle$ for the timestep of interest of sim19. The meaning of the colors is the same as in Fig. C.3. Upper panels: all gas particles within the geometrically defined disk and tidal tail regions. Lower panels: all gas particles within the geometrically defined bridge region.

dynamical model. Equation (C.8) implies that these quantities approximately correspond to their values at the turbulent driving lengthscale. Within an unperturbed galactic disk the driving lengthscale is $l_{\text {driv }}=v_{\text {turb }} t_{\text {turb }} \sim 100 \mathrm{pc}$ and $30 \mathrm{pc}$ at densities of $n \sim 1 \mathrm{~cm}^{-3}$ and $10 \mathrm{~cm}^{-3}$, respectively. These values are broadly consistent with (i) the length scale at which Elmegreen et al. (2003) observed a break in the Fourier transform power spectrum of azimuthal optical and HI intensity scans and (ii) the vertical thickness of the Galactic cold neutral medium (Wolfire et al. 2003). The driving length in the bridge is estimated in Sect. 5. Using Eq. (C.7) instead of Eq. (C.8) leads to equivalent numbers 
of bridge clouds affected by adiabatic compression at the time of interest (today).

The timescales $t_{\text {diss }}$ (Eq. (C.8)) and $t_{\text {comp }}$ (Eq. 1) are important to identify the primary source of energy loss. If $t_{\text {diss }}$ is shorter than $t_{\text {comp }}$, then the dominant energy injection mechanism is star formation and cloud-scale dissipation is more important than adiabatic compression. This is true for galactic disks (Vollmer \& Beckert 2003).

The compression timescale was calculated using the continuity equation

$\frac{\mathrm{d} \rho}{\mathrm{d} t}+\nabla \cdot(\rho v)=0$

All quantities that are needed to derive $t_{\text {comp }}$ and $t_{\text {diss }}$ are calculated from the dynamical model via a Smoothed-Particle Hydrodynamics (SPH)-type algorithm involving the 50 nearest neighbouring particles.

\section{C.5. Star formation suppression caused by turbulent adiabatic compression}

It is generally assumed that within the disks of isolated galaxies turbulence is driven by energy injection through stellar feedback (SN explosions). In an equilibrium state a balance between turbulent pressure and gravity is reached leading to a global virial equilibrium state of the GMCs (Heyer et al. 2009). If the energy injection through large-scale gas compression exceeds that of stellar feedback deduced via the SFR, the velocity dispersion of the largest eddies is expected to increase. In this case, we presume that the velocity dispersion of the turbulent substructures or clouds also increases (Fig. 2 of Mandal et al. 2020). In our toy model, we decided to suppress star formation during a cloud-cloud collision if the energy injection by large-scale gas compression exceeds that from stellar feedback expected from an ISM that forms stars according to a Kennicutt-Schmidt law. For the latter case, the turbulent energy dissipation timescale $t_{\text {diss }}$ can be calculated via our analytical model.

We included the effect of star formation suppression by turbulent adiabatic compression in the following way: If for a cloud-cloud collision $t_{\text {comp }}>0$ and $t_{\text {comp }}<t_{\text {diss }}$, no stellar particle is created. In addition, rapid expansion also suppresses star formation $\left(\left|t_{\text {comp }}\right|<t_{\text {diss }} / 5\right)$. The factor $1 / 5$ was derived heuristically. It allowed us to clearly separate the bridge from the disk regions. This second criterion does not play a dominant role for the outcome of the star formation suppression. We verified that in a simulation of an isolated spiral galaxy this is only the case for a negligible fraction of the gas clouds. Until a few megayears after impact a significant portion of the shocked gas will be hot (Yeager \& Struck 2019) and will not be able to form stars. Since the compression timescale is extremely short, our star formation suppression recipe ensures the absence of star formation in the hot gas.

\section{C.6. Suppressed star formation in the Taffy bridge}

We calculated the SFR within our simulations using the cloudcloud collisions as described in Sect. 5. In the following, we separate the bridge region from the disk regions based on geometry and the gas density. These conditions appear appropriate based on examining the separation in three dimensions. Figure C.5 shows the gas mass in the model bridges. The total gas masses range between $10^{9} M_{\odot}$ for sim19fast to almost $3 \times 10^{9} M_{\odot}$ for sim19.
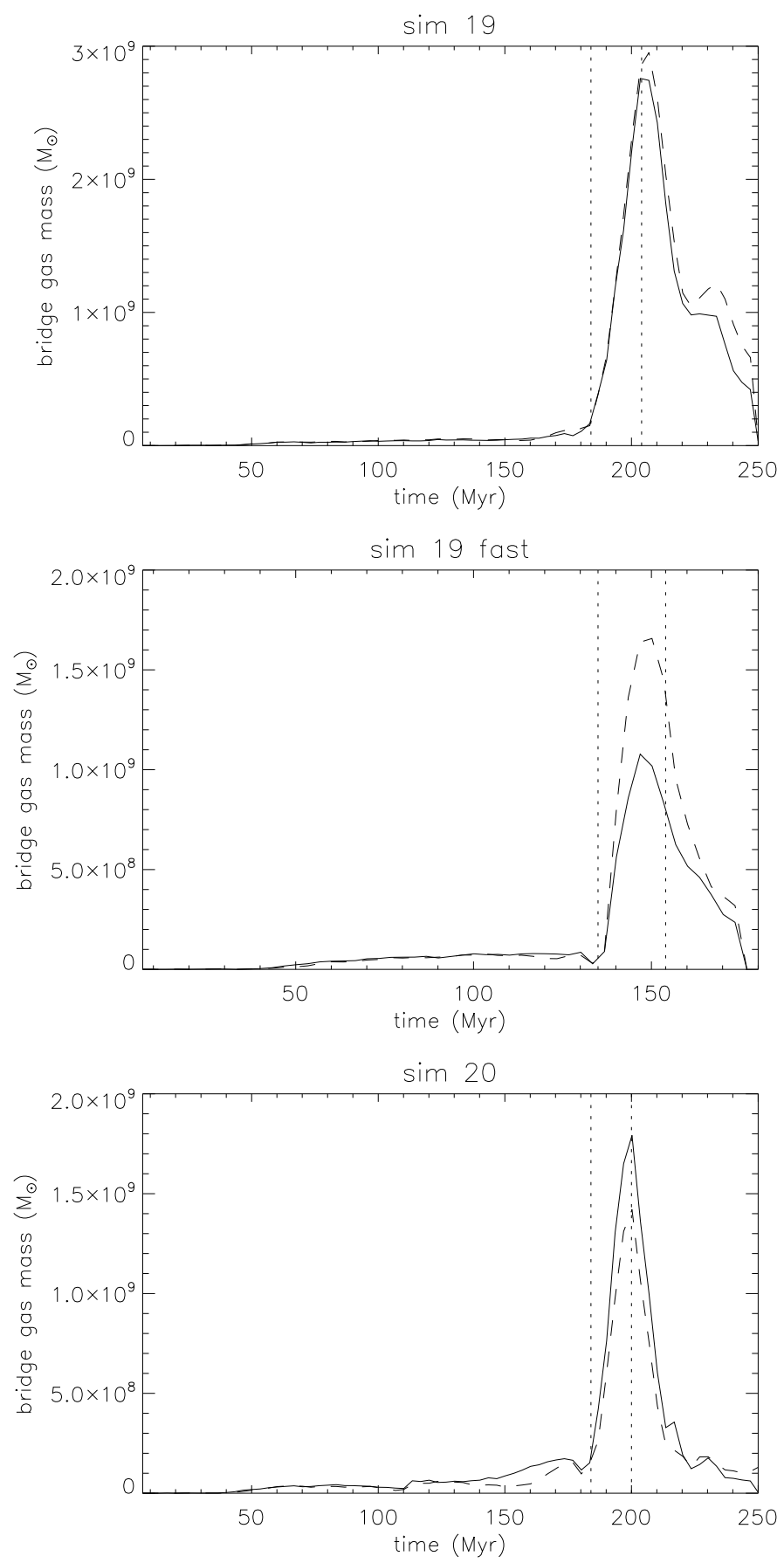

Fig. C.5. Evolution of the total gas mass in the bridge. The dotted vertical lines mark the impact time and the time of interest (today).

The total (disk and bridge) SFR is shown in Fig. C.6 for all three models. It is constant during about $2 / 3$ of the evolution of the system. The evolution of the total SFR of the models without adiabatic compression is much different from that of the models with adiabatic compression. The SFR within the bridge region is shown in Fig. C.7. The comparison between Figs. C.6 and C.7 shows that the strong increase in the total SFR is caused by the star formation in the bridge region. Without adiabatic compression the SFR rapidly increases by a factor of three, whereas the SFR in the models with adiabatic compression stays constant or slowly declines. Our conditions for turbulent adiabatic compression therefore efficiently suppresses star formation in the bridge gas by a factor of $3-5$. 

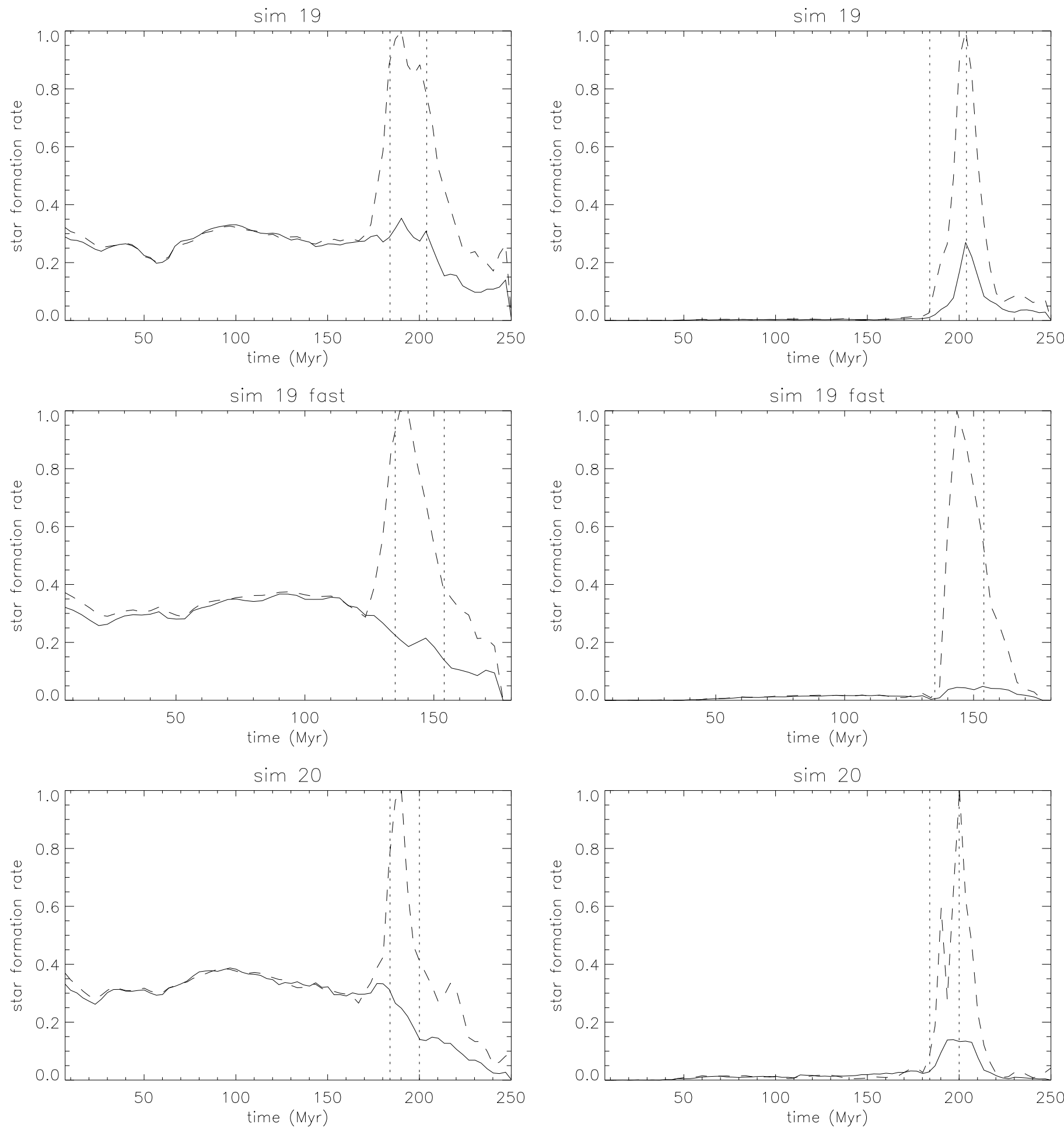

Fig. C.6. Evolution of the normalized total SFR. The solid and dashed lines show evolution with and without turbulent adiabatic compression, respectively. The dotted vertical lines mark the impact time and the time of interest (today).

To illustrate the effect of turbulent adiabatic compression we separated the star-forming and non-star-forming gas particles for the times of interest of the three simulations (Fig. C.8). This shows that our conditions cleanly separate the clouds in the disk and bridge regions. It is worth noting that turbulent adiabatic compression affects gas particles of all volume and column densities.

Fig. C.7. Evolution of the normalized total SFR in the bridge. The solid and dashed lines show evolution with and without turbulent adiabatic compression, respectively. The dotted vertical lines mark the impact time and the time of interest (today).

In all models there are about $30-60 \%$ more particles affected by turbulent adiabatic compression than by rapid expansion. The distributions of these particles for the models are shown in Fig. C.9.

The resulting FUV emission maps based on the models including turbulent adiabatic compression together with the observed GALEX FUV map are presented in Fig. C.10. As 
B. Vollmer et al.: Low star formation efficiency due to turbulent adiabatic compression in the Taffy bridge
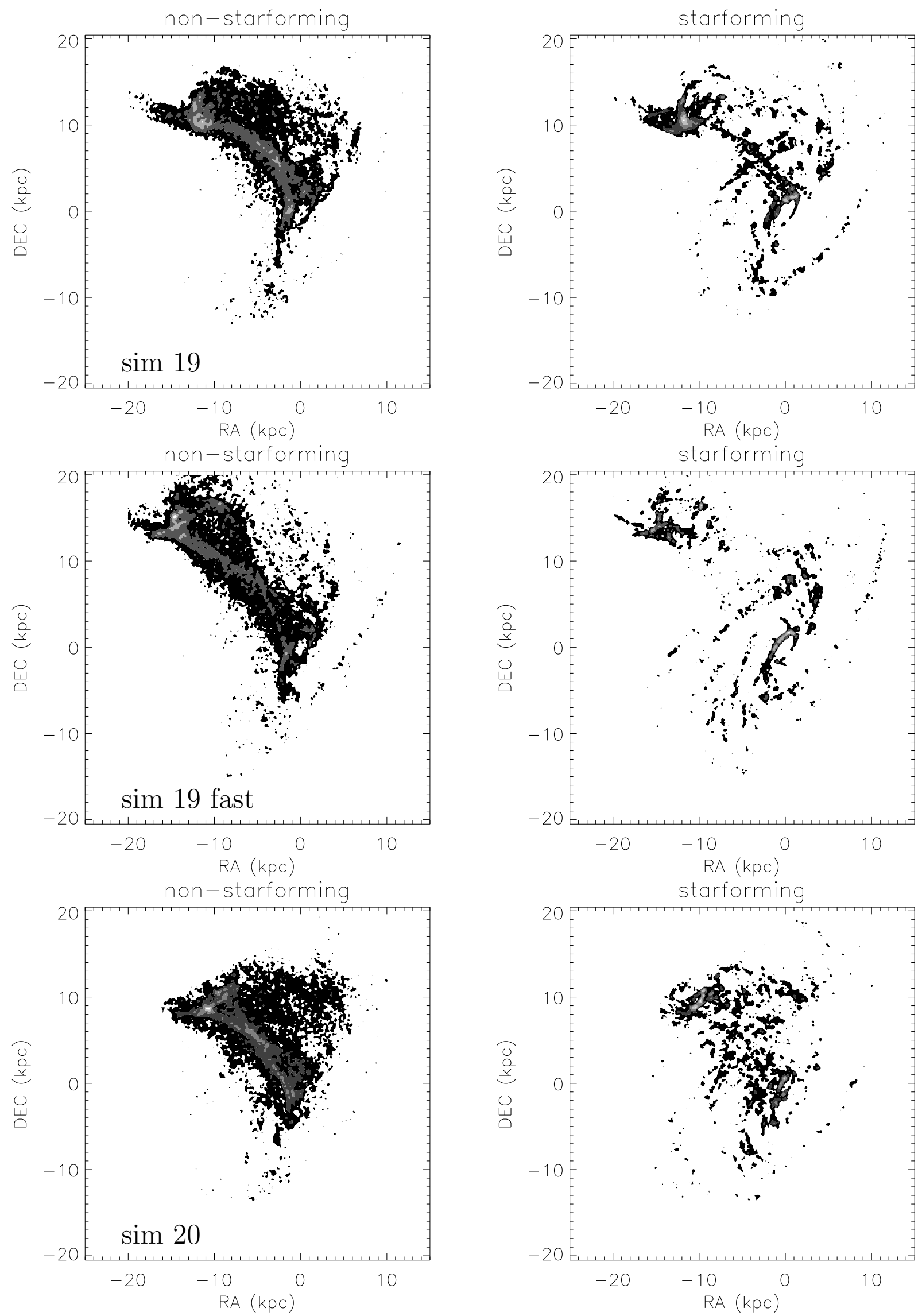

Fig. C.8. Model maps of non-star-forming and star-forming gas. 

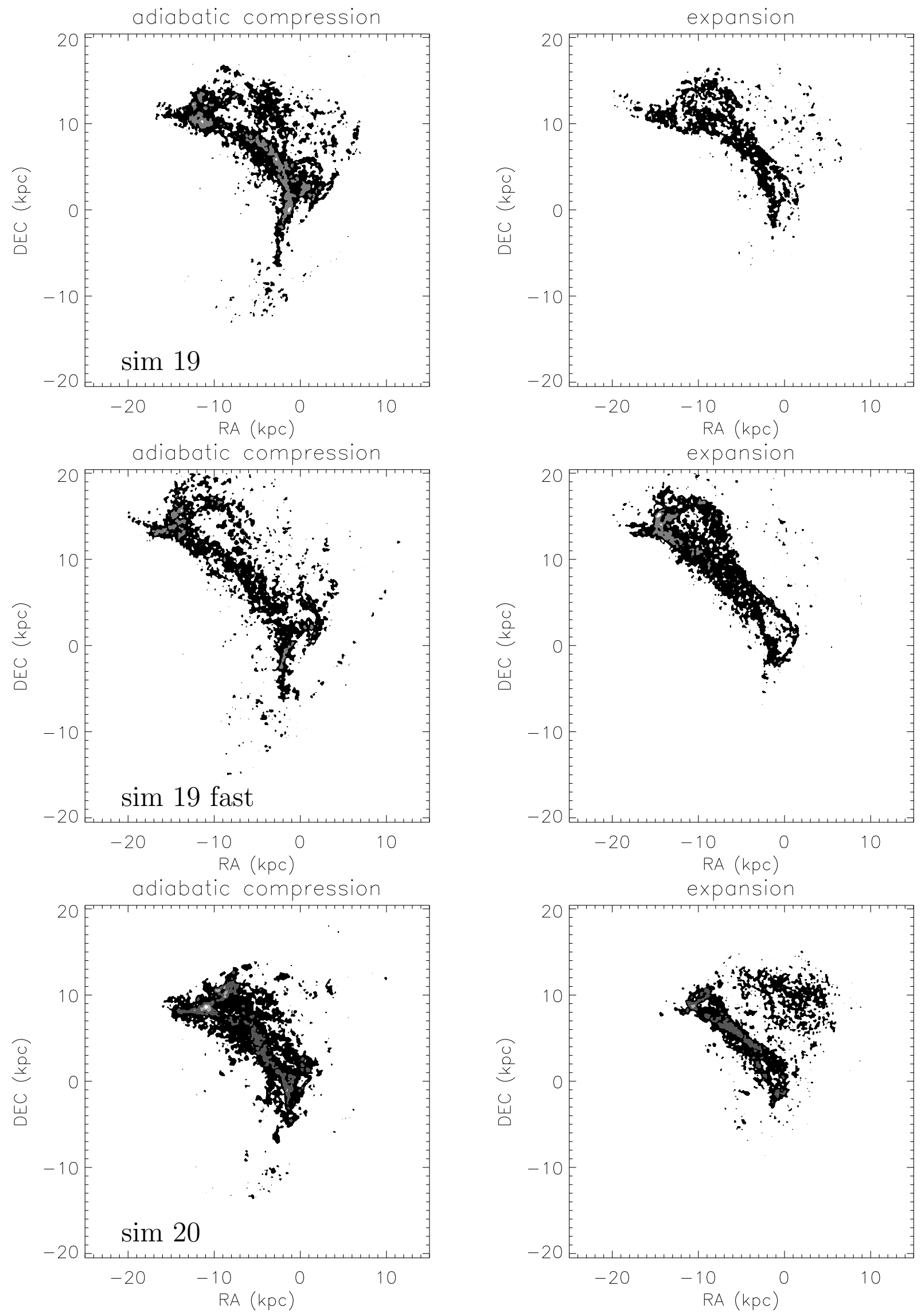

Fig. C.9. Model maps of gas affected by turbulent adiabatic compression and rapid expansion. 
B. Vollmer et al.: Low star formation efficiency due to turbulent adiabatic compression in the Taffy bridge
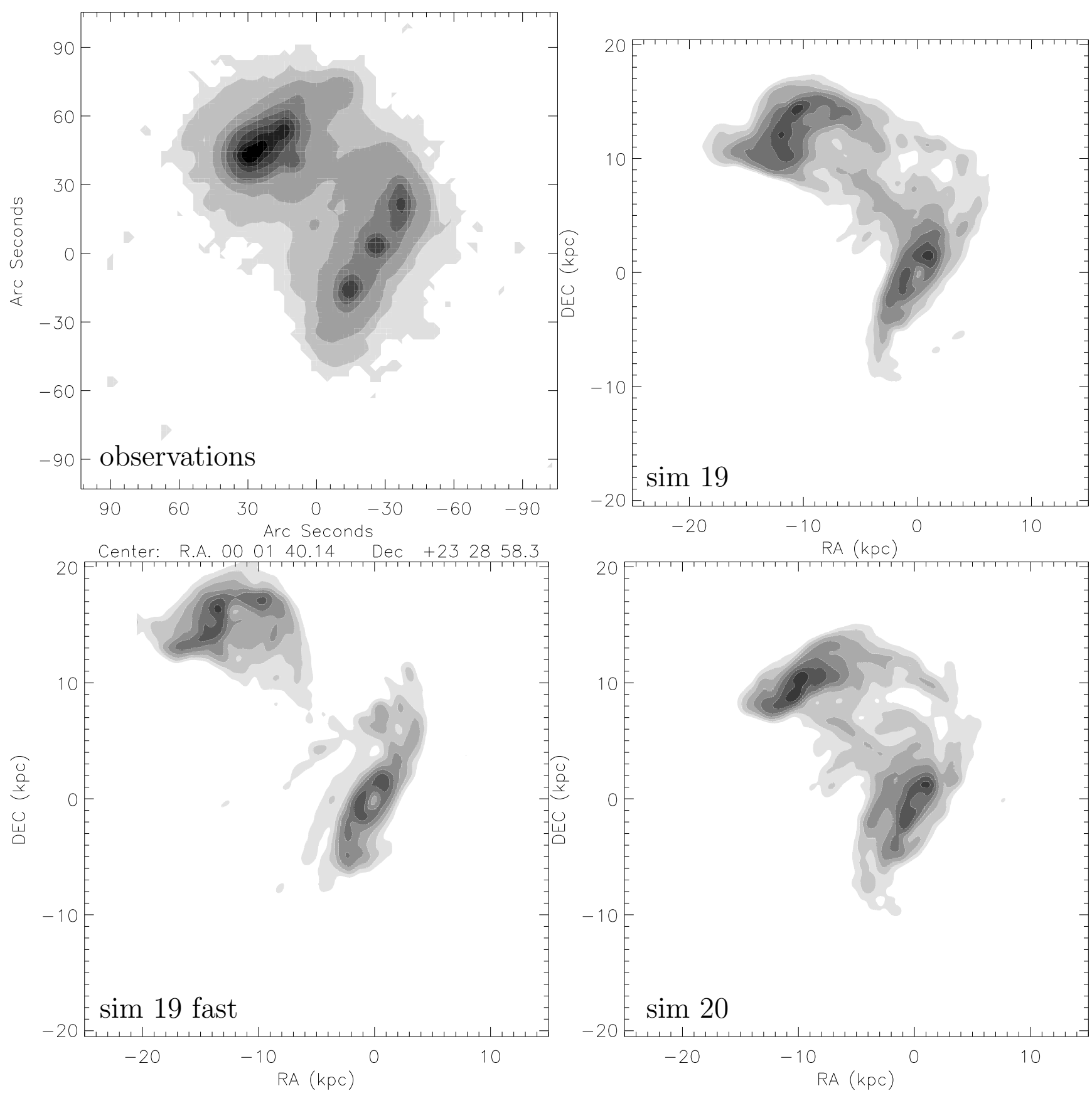

Fig. C.10. Observed star formation map based on Spitzer $24 \mu \mathrm{m}$ and GALEX FUV maps together with the model star formation maps.

expected, the morphology of the FUV emission is very similar to that of the Spitzer $8 \mu \mathrm{m}$ emission (Fig. 3). The corresponding maps from the models without turbulent adiabatic compression are shown in Fig. C.11. The GALEX FUV image does not show structures whose morphology resembles that of the $\mathrm{CO}$ emission with the exception of the compact star formation region close to UGC 12915. The FUV images of sim19 and $\operatorname{sim} 20$ still show some trace of the dense bridge gas. Overall, sim19fast most resembles the GALEX UV image: The emission UGC 12914 and the bridge region are well reproduced. However, as for the gas distribution, the model northern bridge filament is not present in the observations.
The relations between the model SFR and the molecular gas surface density of the three models including adiabatic gas compression are presented in Fig. C.12. Figure C.13 shows the star formation efficiency $\left(\mathrm{SFE}=\mathrm{SFR} / M_{\mathrm{H}_{2}}\right)$ of models 19 and 20 without adiabatic gas compression. The SFE is approximately constant and the gas located in the bridge has only a marginally lower (0.1 dex) SFE than the disks.

In the models with adiabatic gas compression the bridge SFE is $\sim 3$ times lower whereas the disk SFE remains the same. This is comparable to the observed decrease of the star formation efficiency in the Taffy bridge region (Fig. 21 of Vollmer et al. 2012a). 
A\&A 647, A138 (2021)
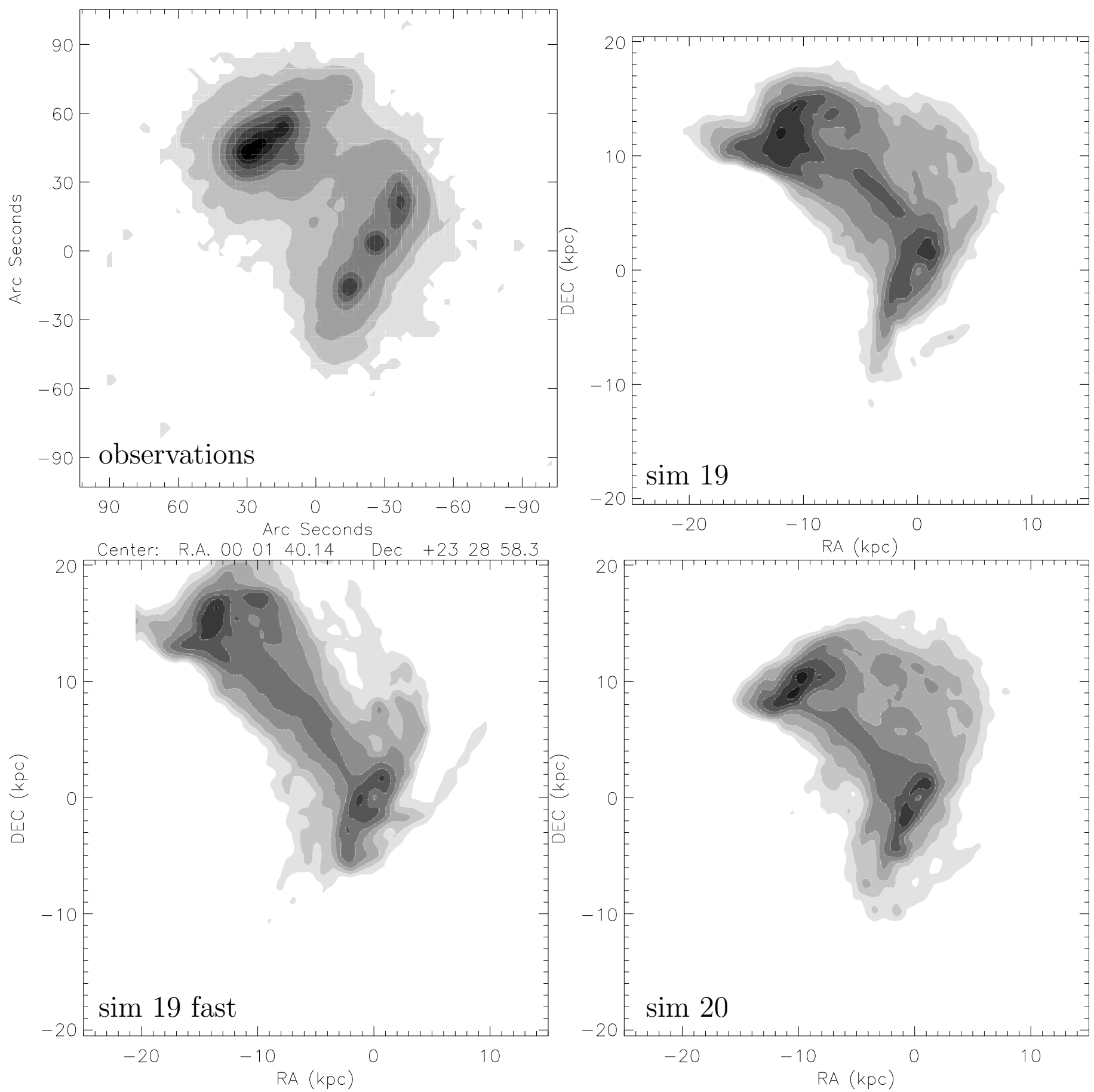

Fig. C.11. Observed star formation map based on Spitzer $24 \mu \mathrm{m}$ and GALEX FUV maps together with the model star formation maps without the suppression of star formation by turbulent adiabatic compression. 
B. Vollmer et al.: Low star formation efficiency due to turbulent adiabatic compression in the Taffy bridge
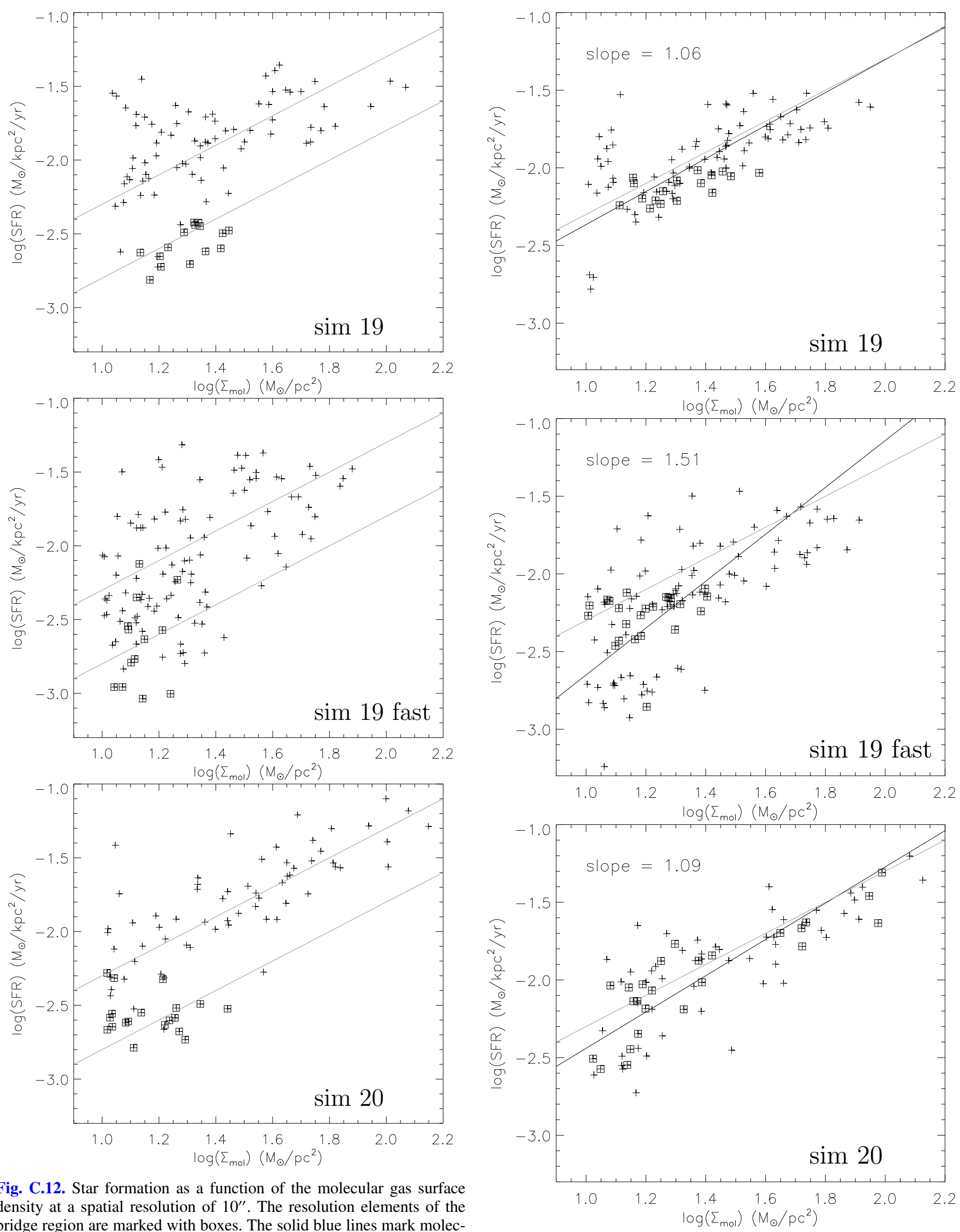

Fig. C.12. Star formation as a function of the molecular gas surface density at a spatial resolution of $10^{\prime \prime}$. The resolution elements of the bridge region are marked with boxes. The solid blue lines mark molecular depletion times of 1.6 and $5 \mathrm{Gyr}$.

Fig. C.13. Same as Fig. C.12 but for the models without adiabatic gas compression. A robust linear bisector fit is shown as a black solid line. 